\title{
Supply Chain Network Design under Demand Uncertainty and Supply Disruptions: A Distributionally Robust Optimization Approach
}

\author{
Ruozhen Qiu and Yizhi Wang \\ School of Business Administration, Northeastern University, No. 195, Chuangxin Road, Hunan New District, \\ Shenyang, Liaoning 110169, China
}

Correspondence should be addressed to Ruozhen Qiu; rzqiu@mail.neu.edu.cn

Received 24 May 2016; Revised 6 August 2016; Accepted 22 September 2016

Academic Editor: Xiaofeng Xu

Copyright (C) 2016 R. Qiu and Y. Wang. This is an open access article distributed under the Creative Commons Attribution License, which permits unrestricted use, distribution, and reproduction in any medium, provided the original work is properly cited.

\begin{abstract}
We develop a robust optimization model for designing a three-echelon supply chain network that consists of manufacturers, distribution centers, and retailers under both demand uncertainty and supply disruptions. The market demands are assumed to be random variables with known distribution and the supply disruptions caused by some of the facilities faults or connection links interruptions are formulated by several scenarios with unknown occurrence probabilities. In particular, we assume the probabilities that the disruption scenarios happen belong to the two predefined uncertainty sets, named box and ellipsoid uncertainty sets, respectively. Through mathematical deductions, the proposed robust SCN design models can be transformed into the tractable linear program for box uncertainty and into second-order cone program for ellipsoid uncertainty. We further offer propositions with proof to show the equivalence of the transformed problems with the original ones. The applications of the proposed models together with solution approaches are investigated in a real case to design a tea supply chain network and validate their effectiveness. Numerical results obtained from model implementation and sensitivity analysis arrive at important practical insights.
\end{abstract}

\section{Introduction}

Designing and managing a supply chain network have become crucial due to the increasing market competition, variable customer demands, and the fast development of the economic and technological globalization. An efficient supply chain network will contribute to quickly responding to the customers' demands and achieving the success of the supply chain which depends on the cooperation and coordination among all members. Supply chain network (SCN) design incorporates both strategic and tactical decisions on the number, location, capacity, and mission of the supply, production, and distribution facilities required to provide goods to a customer base [1]. In recent years, lots of mathematical models have been developed to solve various supply chain network design problems. A. Nagurney and L. S. Nagurney [2] consider a firm that is engaged in determining the capacities of its various supply chain activities and develop a rigorous modelling and analytical framework for the design of sustainable supply chain networks. Bashiri et al. [3] present a mathematical model for strategic and tactical planning in a multiple-echelon, multiple-commodity production-distribution network. Mahdi et al. [4] indicate the SCN design can significantly affect the economic viability of a biofuel technology and develop a mixed-integer linear programming to determine the optimal supply chain design and operation under uncertain environment. Jeihoonian et al. [5] adopt a mixed-integer programming model to design a closed-loop supply chain network for durable goods and coordinate forward flow and reverse flow to determine the position of each type of facilities in the entire network. For comprehensive reviews of SCN design models, see Klibi et al. [6], Tokman and Beitelspacher [7], and Farahani et al. [8].

By investigating the supply chain models, Melo et al. [9] find that both strategic and tactical decisions can be highly affected by various sources of uncertainty such as demand and supply interruptions, lead time variability, exchange rate volatility, and capacity variations [10]. From the perspective 
of risk management, Tang [11] defines two categories of risks in supply chains. One is operational risks arising from business-as-usual incidents, such as machine breakdowns and power outages which lead to uncertainties in matching supply and demand. The second category is disruption risks, which arise from natural and man-made disasters such as earthquakes, floods, hurricanes, and terrorist attacks. Operational risks are usually captured through incorporating such inherent uncertainties in the input data such as uncertain customer demand, uncertain supply capacity, and uncertain costs due to dynamic and fluctuating nature of these parameters. Disruption risks are usually captured by incorporating disruption scenarios in the model formulation of underlying decision problem since they are unlikely to occur but have a high impact when they do occur [12]. To cope with the disadvantage effect of the uncertainties on supply chain operations, Klibi et al. [6] suggest that stochastic programming can be used as a powerful technique to tackle uncertainties. Furthermore, a so-called scenario-based stochastic programming has been paid more attention in SCN design problems. Scenario-based stochastic programming optimizes the expected value of the objective functions by considering a set of discrete scenarios and their occurrence probabilities for random variables. Following such logic, Mulvey et al. [13] initially propose a scenario-based robust stochastic optimization for large-scale systems and define two measures of robustness, namely, model robustness and solution robustness, respectively. A solution to an optimization model is defined as robust solution if it remains close to optimal for all scenarios of the input data and robust model if it remains almost feasible for all data scenarios. By using a parameter reflecting the decision-maker's preference between the solution and model robustness, it incorporates the conflicting objectives of the two. The scenario-based robust optimization can be regarded as a two-stage stochastic programming in essence and has been adopted as an effective tool for designing and managing the supply chain operating in uncertain environments.

Using the robust optimization approach proposed by Mulvey et al. [13], Babazadeh and Razmi [14] develop an efficient mixed-integer linear programming to handle both operational and disruption risks of the agile supply chain network. Baghalian et al. [15] consider both demand-side and supply-side uncertainties and develop a stochastic mathematical formulation for designing a network of multiproduct supply chains. Jabbarzadeh et al. [16] present a dynamic supply chain network design model for the supply of blood during and after disasters which can assist in blood facility location and allocation decisions for multiple postdisaster periods. Jeong et al. [17] provide an integrated framework to design emergency logistics networks comprising distribution warehouses, disaster recovery centers, and neighbourhood locations based on efficiency, risk, and robustness metrics, where the efficiency and risk are determined using transportation cost and relief item loss cost, while the robustness is analyzed in terms of perturbed transportation cost through diverse damage scenarios to major facilities. Fattahi and Govindan [18] apply a two-stage stochastic program to address a multistage and multiperiod supply chain network design problem under stochastic and highly variable demands.

Applications of robust stochastic programming techniques for SCN design models are limited because of the shortage of historical data for fitting probability distributions for uncertain parameters [19]. According to Klibi et al. [6], stochastic programming techniques usually require the perfect information of probability distributions of random variables, such as the likelihood of an interruption occurrence and its magnitude of impact. Such historical data, especially for those rare events, is limited or nonexistent making it difficult or impossible to estimate the actual distribution of uncertain parameters [20]. A robust optimization approach which depends on the complete distributions of the random variables on no condition could be adopted to handle uncertain parameters. Soyster [21] was the first to introduce a robust optimization formulation with interval data uncertainty, which will lead to the overconservative solutions because the probability at which uncertain parameters reach their worst values is as low as that at which they reach their normal values. Subsequently, Ben-Tal and Nemirovski [22], Bertsimas and Sim [23], and Ben-Tal et al. [24, 25] reduce the conservation of Soyster's approach by well defining the uncertainty sets to which the uncertain parameters belong and can thus be applied to design the robust supply chain network. Pishvaee et al. [26] assume the demand and the returns of used products vary in a specified closed bounded box and propose a robust optimization model for handling the inherent uncertainty of input data in a closed-loop supply chain network design problem. Hasani et al. [19] propose a general comprehensive model for strategic closed-loop supply chain network design, in which the uncertainties of parameters such as demand and purchasing cost in the proposed model are handled via an interval robust optimization technique. Hatefi and Jolai [27] propose a robust and reliable model for an integrated forward-reverse logistics network design, which simultaneously takes uncertain parameters and facility disruptions into account. More recently, Hasani et al. [28] propose a robust optimization model based on the uncertainty budget concept to consider uncertain parameters in global supply chain network design. Akbari and Karimi [29] use the similar robust optimization approach to design a multiechelon, multiproduct, multiperiod supply chain network under process uncertainty. Hasani and Khosrojerdi [30] develop a mixed-integer, nonlinear model and consider six flexible and resilience strategies simultaneously in designing robust global supply chain networks under disruptions and uncertainties. In addition, they present an efficient parallel Taguchi-based memetic algorithm to solve the proposed model.

From the literatures mentioned above, it can be seen that the disruption issues have drawn attention in SCN design problems. However, both demand uncertainty and supply disruption are rarely considered simultaneously [15, 31], especially for the unknown disruption occurrence probability. In this paper, we develop a path-based three-echelon robust SCN design model under both demand and supply uncertainties. For the uncertain market demand, we assume, without the loss of generality, it is a random variable with 
a known distribution such as normal distribution, which may lead to a nonlinear model. To improve the solving efficiency, a piecewise linearization method is applied to transform the nonlinear terms into the linear ones. For the supply uncertainty, we consider some supply disruption scenarios caused by the manufacturers' facilities faults or the path interruption between the supply chain nodes. In particular, we use two predefined uncertainty sets, named box and ellipsoid, respectively, to describe the unknown probabilities that the disruption scenarios happen, which motivates us to adopt the robust optimization to build the SCN design models. Furthermore, through mathematical operations, all formulated robust optimization models are transformed into tractable convex programs and can thus be solved efficiently.

The remainder of this paper is organized as follows. We briefly introduce the SCN design problem under demand uncertainty and supply disruption in Section 2 and then propose a SCN design model in Section 3. In Section 4, we proposed two robust SCN design models when the disruption scenarios probabilities are bounded in the box uncertainty set and ellipsoid uncertainty set, respectively. We further transform them into the tractable linear and second-order cone programs to solve. A case study is given in Section 5 to illustrate the proposed models and solutions and to confirm their effectiveness. In the last section, we summarize the paper and discuss future research directions.

\section{Problem Description}

In this paper, we focus on the SCN design of a threeechelon supply chain under both the demand uncertainty and supply disruptions. The supply chain involves some potential manufactures, distribution centers, and retailers located in some candidate locations of the markets. As the market terminals, the retailers are in charge of the selling and face uncertain market demands that are considered as random variables with known distribution. Before observing the market demands, retailers need to place orders from the upstream. The distribution centers integrate the orders from the retailers and pass them to the manufacturers. To achieve economies of scale, the manufactures are inclined to integrate the orders and then organize production or purchase from the outer suppliers. Once the goods are produced and ready for shipment, they will be transported to distribution centers by consolidation and then arranged for delivery to multiple destinations. To meet the requirement of each market, a cross-dock operation can be adopted in the distribution centers to replenish fast-moving store inventories.

Figure 1 depicts the basic network structure. We also use the concept of a path or route instead of defining flow variables among the nodes of the supply chain [15]. Thus, each link starting from a manufacturer, passing through a distribution center, and ending at a retailer can be regarded as a potential transportation route. We further consider disruption risks in supply chain which impose great impacts on the companies' performance. According to Azad et al. [32], sources of disruption risks can be segmented into two categories: random disruption risks which may occur at any

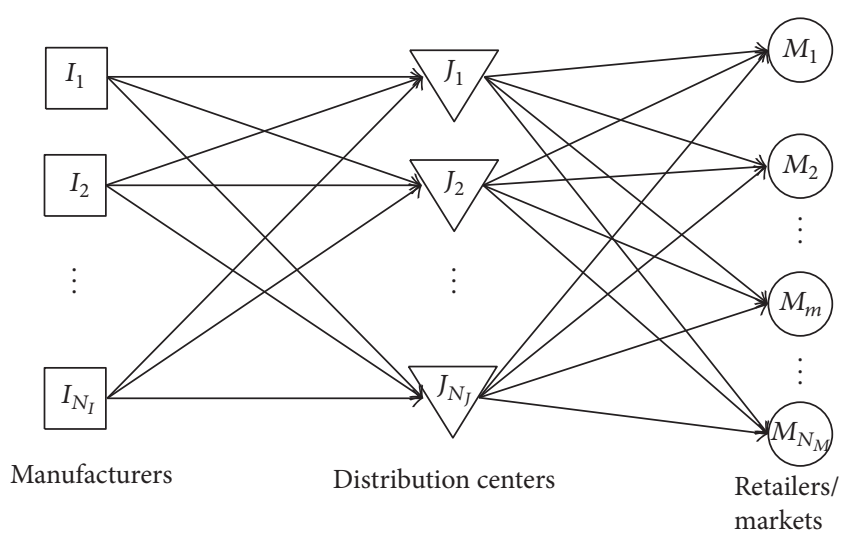

FIGURE 1: The three-echelon supply chain network.

physical point of supply chain network, for example, natural hazards and earthquakes, and premeditated disruption risks which are deliberately planned to inflict the supply chain with maximum damages.

In this paper, we mainly aim to explore the SCN design under premeditated disruption risks. In particular, we consider the supply disruptions due to the manufacturers' facilities impairments or connection links interruptions which result in some of the potential routes being unusable. The disruption set caused by a specific event is called a scenario and is assigned with a finite but unknown probability of occurrence, which is remarkably different from the literatures with the assumption of knowing the scenarios occurrence probability.

We propose a robust SCN design model incorporating both the demand uncertainty and supply disruptions to determine supply chain networks and operation strategies that can perform well under all scenarios. Two types of decision variables are considered in the proposed model. One involves numbers and locations of the distribution centers or retailers and is named the first-stage decision variables. The other concerns the product flow along the different routes and is named the second-stage decision variables. The former are used to determine the network structure, while the latter match with the operation strategies. Thus, the proposed robust SCN design models can be considered as two-stage robust optimization problems.

\section{Mathematical Formulations}

The sets, parameters, and variables used in the formulation of the robust SCN design model are defined in the Notations.

The expected profit of each market $m$ in scenario $s$ is given by

$$
\begin{gathered}
E\left[\pi_{s m}\right]=E\left[\sum_{p \in P} P_{m p} \cdot \min \left\{\sum_{r \in R^{m}} Q_{p s r}, D_{m p}\right\}\right. \\
+\sum_{p \in P} V_{m p} \cdot\left(\sum_{r \in R^{m}} Q_{p s r}-D_{m p}\right)^{+}-\sum_{p \in P} C_{m p}
\end{gathered}
$$




$$
\begin{aligned}
& \left.\cdot\left(D_{m p}-\sum_{r \in R^{m}} Q_{p s r}\right)^{+}-\sum_{p \in P} \sum_{r \in R^{m}} H_{p r} \cdot Q_{p s r}\right] \\
& =\sum_{p \in P}\left(P_{m p}+C_{m p}\right) \sum_{r \in R^{m}} Q_{p s r} \\
& -\sum_{p \in P}\left(P_{m p}+C_{m p}-V_{m p}\right) \int_{0}^{\sum_{r \in R^{m}} Q_{p s r}} F\left(D_{m p}\right) d D_{m p} \\
& -\sum_{p \in P} C_{m p} \cdot E\left[D_{m p}\right] \cdot \zeta_{m}-\sum_{p \in P} \sum_{r \in R^{m}} H_{p r} \cdot Q_{p s r},
\end{aligned}
$$

where $x^{+}=\max \{x, 0\}$ and $E[\cdot]$ represents the expectation operator. Note that the supply disruptions caused by the impairments of the manufacturer's facilities or the interruptions of the connection links are taken into account by defining several scenarios. In each scenario, some of the potential routes are unavailable. Therefore, the SCN design model incorporating the demand uncertainty and supply disruptions can be described as

$\max \Pi$

$$
\begin{array}{ll} 
& =\sum_{s \in S} p_{s}\left(\sum_{m \in M} \sum_{p \in P}\left(P_{m p}+C_{m p}\right) \sum_{r \in R^{m}} Q_{p s r}-\sum_{m \in M} \sum_{p \in P}\left(P_{m p}+C_{m p}-V_{m p}\right) \int_{0}^{\sum_{r \in R^{m}} Q_{p s r}} F\left(D_{m p}\right) d D_{m p}-\sum_{m \in M} \sum_{p \in P} C_{m p}\right. \\
& \left.\cdot E\left[D_{m p}\right] \cdot \zeta_{m}-\sum_{p \in P} \sum_{r \in R^{m}} H_{p r} \cdot Q_{p s r}\right)-\sum_{j \in J} G_{j} \cdot \kappa_{j}-\sum_{m \in M} L_{m} \cdot \zeta_{m} \\
\text { s.t. } \quad & \sum_{p \in P} Q_{p s r} \leq W \cdot B_{s r}, \quad \forall s \in S, r \in R, \\
& \sum_{p \in P} \sum_{r \in R} Q_{p s r} \leq W \cdot \kappa_{j}, \quad \forall s \in S, j \in J, \\
& \sum_{p \in P} \sum_{r \in R} Q_{p s r} \leq W \cdot \zeta_{m}, \quad \forall s \in S, m \in M, \\
& \sum_{j \in J} G_{j} \cdot \kappa_{j}+\sum_{m \in M} L_{m} \cdot \zeta_{m} \leq y, \\
& \sum_{r \in R^{i}} Q_{p s r} \leq Q_{i p}^{\max }, \quad \forall i \in I, p \in P, s \in S, \\
& Q_{p s r} \geq 0, \quad \forall p \in P, s \in S, r \in R, \\
& \kappa_{j}, \zeta_{m} \in\{0,1\}, \quad \forall j \in J, m \in M .
\end{array}
$$$$
\left.\cdot E\left[D_{m p}\right] \cdot \zeta_{m}-\sum_{p \in P} \sum_{r \in R^{m}} H_{p r} \cdot Q_{p s r}\right)-\sum_{j \in J} G_{j} \cdot \kappa_{j}-\sum_{m \in M} L_{m} \cdot \zeta_{m}
$$

Objective function (2) aims to maximize the whole supply chain network's expected profit under all the scenarios. Constraint (3) represents that the products can only be distributed along the feasible routes in the network in each scenario $s$, where $W$ is a given big positive. Constraints (4) and (5) impose the capacity restrictions on the located facilities. Constraint (6) implies that the cost expense of locating the facilities in the network cannot exceed the budget. Constraint (7) imposes the amount of product $p$ shipped along route $r \in R^{i}$ that should be less than the maximum capacity of manufacturer $i$. Constraint (8) means the product flow in the network is positive. Constraint (9) includes two binary variables used to decide whether a facility should be established.
Problems (2)-(9) cannot be solved efficiently due to the nonlinear term $\int_{0}^{\sum_{r \in R^{m}} X_{p s r}} F\left(D_{m p}\right) d D_{m p}$ in (2). For example, if the demand follows a normal distribution, calculating this term is not straightforward since the integral of cumulative distribution function cannot be a closed form. We use a piecewise linear transformation to convert the nonlinear function into a linear one in each interval divided by golden section method. The linearized function is not exactly the same as the original nonlinear one, which will result in an error between the two. However, the error can be decreased by increasing the number of intervals. Figure 2 shows the linearization approximation process of continuous nonlinear function $f(x)\left(x \in\left[x_{l}, x_{u}\right], L=x_{u}-x_{l}\right)$. Let $x_{1}=x_{l}$ 


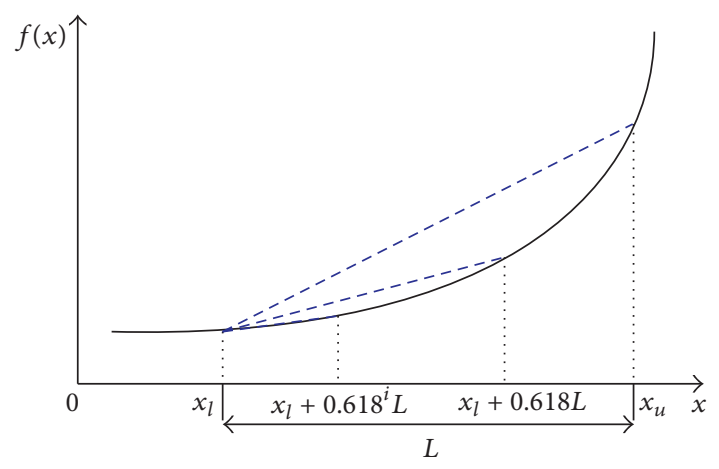

FIGURE 2: Piecewise linearization of a nonlinear function.

and $y_{1}=f\left(x_{1}\right)$; we then obtain the first golden section ratio $x_{2}=x_{1}+0.618 \cdot L$ corresponding to a function value $y_{2}$ and a linear equation $y=k\left(x-x_{1}\right)+y_{1}$ with slope $k=\left(y_{2}-y_{1}\right) /\left(x_{2}-\right.$ $\left.x_{1}\right)=\left(y_{2}-y_{1}\right) /(0.618 \cdot L)$ on the interval $\left[x_{1}, x_{2}\right]$. Similarly, we have the $i$ th golden section ratio $x_{2}=x_{1}+0.618^{i} \cdot L$ when starting from the initial point $x_{1}$ and the corresponding linear equation $y=k_{i}\left(x-x_{1}\right)+y_{1}$ with the slope $k_{i}=\left(y_{2}-y_{1}\right) /\left(x_{2}-\right.$ $\left.x_{1}\right)=\left(y_{2}-y_{1}\right) /\left(0.618^{i} \cdot L\right)$. Consider the fitting error, denoted by $\varepsilon=y-f(x)=k_{i}\left(x-x_{1}\right)+y_{1}-f(x)$. The maximum error $\varepsilon_{\max }$ is achieved at $d \varepsilon / d x=k_{i}-f^{\prime}(x)=0$. The linear equation $y=k_{i}\left(x-x_{1}\right)+y_{1}$ can be used to approximately substitute the nonlinear term on the interval $\left[x_{1}, x_{2}\right]$ if the maximum error is allowable. Otherwise, we can continue to consider $(i+1)$ th golden section ratio. Let $x_{2}$ be an initial point and follow the process above to perform the next linearization until we get the piecewise linear functions on the whole interval $\left[x_{l}, x_{u}\right]$.

Let $n^{m} \in N^{m}=\{1,2, \ldots\}$ signify the set of approximation intervals for each market $m$, and define $a_{n^{m}}$ and $b_{n^{m}}$ as the slope coefficient and intercept of the linear function for interval $n^{m}$ of market $m$, respectively. Besides, $u_{n^{m}}$ and $d_{n^{m}}$ are specified to represent the lower and upper bounds for interval $n^{m}$ of market $m$. The decision variable $Q_{p s r}$ can thus be replaced with $Q_{n^{m} p s r}$ which expresses the amount of product $p$ shipped along route $r$ in scenario $s$ for the interval $n^{m}$. Therefore, the SCD design model (2)-(9) can be reformulated as the following linear program:

$$
\begin{aligned}
& \max \sum_{s \in S} p_{s} \cdot \pi_{s}-\sum_{j \in J} G_{j} \cdot \kappa_{j}-\sum_{m \in M} L_{m} \cdot \zeta_{m} \\
& \text { s.t. } \pi_{s} \\
& =\sum_{m \in M} \sum_{p \in P}\left(P_{m p}+C_{m p}\right) \cdot \sum_{r \in R^{m}} \sum_{n^{m} \in N^{m}} Q_{n^{m}} p s r-\sum_{m \in M} \sum_{p \in P}\left(P_{m p}+C_{m p}-V_{m p}\right) \sum_{n^{m} \in N^{m}}\left(a_{n^{m}} \sum_{r \in R^{m}} Q_{n^{m} p s r}+b_{n^{m}} \theta_{n^{m} p s}\right) \\
& -\sum_{m \in M} \sum_{p \in P} C_{m p} \cdot E\left[D_{m p}\right] \cdot \zeta_{m}-\sum_{p \in P} \sum_{r \in R^{m}} H_{p r} \cdot \sum_{n^{m} \in N^{m}} Q_{n^{m} p s r}, \quad \forall s \in S, \\
& \sum_{m \in M} \sum_{n^{m} \in N^{m}} \sum_{p \in P} \sum_{r \in R^{j}} Q_{n^{m}} p s r \leq W \cdot \kappa_{j}, \quad \forall j \in J, \quad \forall s \in S, \\
& \sum_{m \in M} \sum_{n^{m} \in N^{m}} \sum_{p \in P} \sum_{r \in R^{m}} Q_{n^{m} p s r} \leq W \cdot \zeta_{m}, \quad \forall m \in M, \forall s \in S, \\
& \sum_{m \in M} \sum_{n^{m} \in N^{m}} \sum_{r \in R^{i}} Q_{n^{m}} p s r \leq U_{i p}, \quad \forall i \in I, \forall p \in P, \forall s \in S, \\
& \sum_{j \in J} G_{j} \cdot \kappa_{j}+\sum_{m \in M} L_{m} \cdot \zeta_{m} \leq y, \\
& \sum_{n^{m} \in N^{m}} \theta_{n^{m} p s}=1, \quad \forall p \in P, \forall s \in S, \\
& \sum_{r \in R^{m}} Q_{n^{m} p s r} \leq u_{n^{m}} \cdot \theta_{n^{m} p s}, \quad \forall n^{m} \in N^{m}, \forall s \in S, \forall p \in P, \\
& \sum_{r \in R^{m}} Q_{n^{m} p s r} \geq d_{n^{m}} \cdot \theta_{n^{m} p s}, \quad \forall n^{m} \in N^{m}, \forall s \in S, \forall p \in P, \\
& Q_{n^{m} p s r} \geq 0, \quad \forall n^{m} \in N^{m}, \forall p \in P, \forall s \in S, \forall r \in R, \\
& \theta_{n^{m} p s} \in\{0,1\}, \quad \forall n^{m} \in N^{m}, \forall p \in P, \forall s \in S, \\
& \kappa_{j}, \zeta_{m} \in\{0,1\}, \quad \forall j \in J, \forall m \in M,
\end{aligned}
$$


where $\pi_{s}$ is an auxiliary variable introduced to simplify the model description. Note that the binary variable $\theta_{n^{m} p s}$ is equal to 1 if interval $n^{m}$ is selected for product $p$ in market $m$ under scenario $s$ and 0 otherwise. Constraint (16) ensures that only one interval can be selected for each market. Constraints (17)(18) indicate the amount of product $p$ of each interval is restricted by its boundary. Other constraints are explicated as shown above.

The optimal profit of the whole supply chain in the real scenario may differ from the objective function value derived from the proposed mathematical model; we introduce the solution robustness defined in Mulvey et al. [13] to reduce the deviation and make the SCN design model more robust:

$$
\begin{aligned}
\max & \sum_{s \in S} p_{s} \cdot \pi_{s}-\lambda \sum_{s \in S} p_{s} \cdot\left(\pi_{s}-\sum_{s^{\prime} \in S} p_{s^{\prime}} \cdot \pi_{s^{\prime}}\right)^{2} \\
& -\sum_{j \in J} G_{j} \cdot \kappa_{j}-\sum_{m \in M} L_{m} \cdot \zeta_{m}
\end{aligned}
$$

s.t. constraints $(11)-(21)$,

where $\lambda$ denotes the weight used to measure the solution variance. The objective value of problem (22a) is less sensitive to change in the input data under all scenarios as the variable weight, $\lambda$, increases. However, the quadratic term, $\sum_{s \in S} p_{s}\left(\pi_{s}-\sum_{s^{\prime} \in S} p_{s^{\prime}} \pi_{s^{\prime}}\right)^{2}$, which evaluates the closeness of a solution to optimality for $s \in S$ usually requires a large computation time. Yu and $\mathrm{Li}$ [33] suggest that the quadratic term can be replaced with $\sum_{s \in S} p_{s}\left|\pi_{s}-\sum_{s^{\prime} \in S} p_{s^{\prime}} \pi_{s^{\prime}}\right|$. By the approach proposed in $\mathrm{Yu}$ and $\mathrm{Li}$ [33], problem (22a) can be converted into the following equivalent linear formulation:

$$
\begin{aligned}
\max \sum_{s \in S} p_{s} \cdot \pi_{s}-\lambda \sum_{s \in S} p_{s} \\
\cdot\left[\left(\sum_{s^{\prime} \in S} p_{s^{\prime}} \cdot \pi_{s^{\prime}}-\pi_{s}\right)+2 \omega_{s}\right]-\sum_{j \in J} G_{j} \cdot \kappa_{j} \\
-\sum_{m \in M} L_{m} \cdot \zeta_{m}
\end{aligned}
$$

s.t. $\quad \sum_{s \in S} p_{s} \cdot \pi_{s}-\pi_{s}+\omega_{s} \geq 0, \quad \forall s \in S$,

$\omega_{s} \geq 0, \quad \forall s \in S$,

constraints $(11)-(21)$.

Note that if $\sum_{s^{\prime} \in S} p_{s^{\prime}} \cdot \pi_{s^{\prime}}-\pi_{s} \geq 0 \forall s \in S$, the maximization objective requires $\omega_{s}=0$; otherwise, $\omega_{s}=\pi_{s}-\sum_{s \in S} p_{s} \cdot \pi_{s}$ due to the first constraint of problem (22b). Therefore, the solution of problem (22b) is similar to that of problem (22a) when the quadratic term $\sum_{s \in S} p_{s}\left(\pi_{s}-\sum_{s^{\prime} \in S} p_{s^{\prime}} \pi_{s^{\prime}}\right)^{2}$ is replaced by $\sum_{s \in S} p_{s}\left|\pi_{s}-\sum_{s^{\prime} \in S} p_{s^{\prime}} \pi_{s^{\prime}}\right|$. Obviously, problem (22b) is a linear program and can thus be solved efficiently when the probability of the disruption occurrence of scenario $s \in S$ is known. However, it is difficult or impossible to estimate the actual distribution of uncertain parameters due to the lack of the historical data, especially for those rare events [20]. In the next section, we will further discuss the situation that the accurate probability of supply disruption is unavailable.

\section{Robust SCN Design Model under Uncertain Supply Disruption Probability}

Assume the probability distribution of the supply disruption is not complete and only known to belong to an uncertain set $\mathscr{P}$ with pr $\in \mathscr{P}, \mathbf{p r}=\left(p_{1}, p_{2}, \ldots, p_{N_{\mathrm{S}}}\right)^{T}$. The robust counterpart of problem (22b) can be formulated as

$$
\begin{aligned}
\max _{\boldsymbol{\Theta}, \boldsymbol{\omega}} \min _{\mathbf{p r} \in \mathscr{P}} \sum_{s \in S} p_{s} \cdot \pi_{s}-\lambda \sum_{s \in S} p_{s} \\
\cdot\left[\left(\sum_{s^{\prime} \in S} p_{s^{\prime}} \cdot \pi_{s^{\prime}}-\pi_{s}\right)+2 \omega_{s}\right]-\sum_{j \in J} G_{j} \\
\cdot \kappa_{j}-\sum_{m \in M} L_{m} \cdot \zeta_{m} \\
\text { s.t. } \quad \min _{\mathbf{p r} \in \mathscr{P}} \sum_{s \in S} p_{s} \cdot \pi_{s} \geq \pi_{s}-\omega_{s}, \quad \forall s \in S, \\
\quad \omega_{s} \geq 0, \quad \forall s \in S, \\
\text { constraints }(11)-(21),
\end{aligned}
$$

where $\Theta \in R^{N_{d}}$ denotes the set of the decision variables and $\boldsymbol{\omega}=\left(\omega_{1}, \omega_{2}, \ldots, \omega_{N_{S}}\right)^{T}$ is the auxiliary vector [33]. Note that problem (23) cannot be solved directly due to the min operators in both objective function and the first constraint. In the following, we introduce two uncertainty sets, namely, box and ellipsoid. Problem (23) can thus be transformed into a linear program and a second-order cone program, respectively.

4.1. Robust SCN Design Model under Box Uncertainty. The box uncertainty set to which the probability of the supply disruption scenario belongs is defined as follows:

$$
\mathbf{p r} \in \mathscr{P}_{I} \triangleq\left\{\mathbf{p r} \mid \mathbf{p r}=\overline{\mathbf{p}}+\boldsymbol{\xi}, \mathbf{e}^{T} \boldsymbol{\xi}=0, \underline{\xi} \leq \boldsymbol{\xi} \leq \overline{\boldsymbol{\xi}}\right\},
$$

where $\overline{\mathbf{p}}=\left(\bar{p}_{1}, \bar{p}_{2}, \ldots, \bar{p}_{N_{S}}\right)^{T}$ is a nominal distribution that represents the most likely distribution of the disruption probability, $\boldsymbol{\xi} \in[\boldsymbol{\xi}, \bar{\xi}]$ denotes the uncertain parameter vector, and $\mathbf{e}$ signifies the vector of one. The constraint $\mathbf{e}^{T} \boldsymbol{\xi}=$ 0 ensures pr will meet the requirement of the probability distribution.

Let us first consider the following max-min optimization problem in (24):

$$
\max _{\boldsymbol{\Theta}, \boldsymbol{\omega}} \min _{\mathbf{p r} \in \mathscr{P}_{I}}(\boldsymbol{\pi}-2 \lambda \boldsymbol{\omega})^{T} \mathbf{p r}-\bar{f},
$$

where $\pi=\left(\pi_{1}, \pi_{2}, \ldots, \pi_{N_{S}}\right)^{T}$ and $\bar{f}=\sum_{j \in J} G_{j} \cdot \kappa_{j}+\sum_{m \in M} L_{m}$. $\zeta_{m}$. The inner minimization problem is

$$
\begin{aligned}
\min _{\mathbf{p r} \in \mathscr{P}_{I}} & \left\{(\boldsymbol{\pi}-2 \lambda \boldsymbol{\omega})^{T} \mathbf{p r}-\bar{f}\right\} \\
= & (\boldsymbol{\pi}-2 \lambda \boldsymbol{\omega})^{T} \overline{\mathbf{p}}+\mathscr{G}^{*}(\boldsymbol{\xi})-\bar{f},
\end{aligned}
$$


where $\mathscr{G}^{*}(\xi)$ is the optimal value of the following problem:

$$
\min _{\xi}\left\{(\pi-2 \lambda \omega)^{T} \boldsymbol{\xi} \mid \mathbf{e}^{T} \boldsymbol{\xi}=0, \underline{\xi} \leq \boldsymbol{\xi} \leq \bar{\xi}\right\} .
$$

The dual program of (27) can be described as follows:

$$
\max _{\delta, \boldsymbol{\tau}, \boldsymbol{\nu}}\left\{\overline{\boldsymbol{\xi}}^{T} \boldsymbol{\tau}+\underline{\xi}^{T} \boldsymbol{\nu} \mid \mathrm{e} \delta+\boldsymbol{\tau}+\boldsymbol{\nu}=\boldsymbol{\pi}-2 \lambda \boldsymbol{\omega}, \boldsymbol{\tau} \leq \mathbf{0}, \boldsymbol{\nu} \geq \mathbf{0}\right\} .
$$

According to the linear programming duality theory, it can be seen that the optimal value of the objective function in problem (27) is equal to that in (28). Following the similar mathematic process, the left-hand side of the first constraint in (23) is given by

$$
\begin{aligned}
\min _{\mathbf{p r} \in \mathscr{P}_{I}} & \boldsymbol{\pi}^{T} \mathbf{p r} \\
& =\min _{\boldsymbol{\xi}}\left\{\boldsymbol{\pi}^{T} \overline{\mathbf{p}}+\boldsymbol{\pi}^{T} \boldsymbol{\xi} \mid \mathbf{e}^{T} \boldsymbol{\xi}=0, \underline{\boldsymbol{\xi}} \leq \boldsymbol{\xi} \leq \overline{\boldsymbol{\xi}}\right\} \\
& =\boldsymbol{\pi}^{T} \overline{\mathbf{p}}+\mathscr{R}^{*}(\boldsymbol{\xi}),
\end{aligned}
$$

where $\mathscr{R}^{*}(\boldsymbol{\xi})$ is the optimal value of $\min _{\boldsymbol{\xi}}\left\{\boldsymbol{\pi} \boldsymbol{\xi} \mid \mathrm{e}^{T} \boldsymbol{\xi}=0, \underline{\xi} \leq\right.$ $\boldsymbol{\xi} \leq \bar{\xi}\}$, which has the following dual problem:

$$
\max _{\gamma, \boldsymbol{\alpha}, \boldsymbol{\beta}}\left\{\overline{\boldsymbol{\xi}}^{T} \boldsymbol{\alpha}+\underline{\xi}^{T} \boldsymbol{\beta} \mid \mathbf{e} \gamma+\boldsymbol{\alpha}+\boldsymbol{\beta}=\boldsymbol{\pi}, \boldsymbol{\alpha} \leq \mathbf{0}, \boldsymbol{\beta} \leq \mathbf{0}\right\} .
$$

Now, consider the following optimization problem:

$$
\begin{aligned}
\max _{\substack{\boldsymbol{\Theta}, \boldsymbol{\omega}, \delta, \boldsymbol{\tau} \\
\nu, \gamma, \boldsymbol{\alpha}, \boldsymbol{\beta}}} & (\boldsymbol{\pi}-2 \lambda \boldsymbol{\omega})^{T} \overline{\mathbf{p}}+\overline{\boldsymbol{\xi}}^{T} \boldsymbol{\tau}+\underline{\xi}^{T} \boldsymbol{\nu}-\bar{f} \\
\text { s.t. } & \mathbf{e} \delta+\boldsymbol{\tau}+\boldsymbol{\nu}=\boldsymbol{\pi}-2 \lambda \boldsymbol{\omega}, \\
& \mathbf{e} \gamma+\boldsymbol{\alpha}+\boldsymbol{\beta}=\boldsymbol{\pi}, \\
& \boldsymbol{\pi}^{T} \overline{\mathbf{p}}+\overline{\boldsymbol{\xi}}^{T} \boldsymbol{\alpha}+\underline{\xi}^{T} \boldsymbol{\beta} \geq \pi_{s}-\omega_{s}, \quad \forall s \in S, \\
& \boldsymbol{\tau} \leq \mathbf{0}, \quad \boldsymbol{\nu} \geq \mathbf{0}, \boldsymbol{\alpha} \leq \mathbf{0}, \boldsymbol{\beta} \geq \mathbf{0}, \\
& \omega_{s} \geq 0, \quad \forall s \in S, \\
& \text { constraints }(11)-(21) .
\end{aligned}
$$

Proposition 1 shows that solving (23) under box uncertainty is equivalent to solving (31) with variables $(\boldsymbol{\Theta}, \boldsymbol{\omega}, \delta, \boldsymbol{\tau}, \boldsymbol{\nu}, \boldsymbol{\gamma}, \boldsymbol{\alpha}, \boldsymbol{\beta}) \in R^{N_{d}} \times R \times R^{N_{S}} \times R^{N_{S}} \times R \times R^{N_{S}} \times R^{N_{S}}$.

Proposition 1. If the solution $\left(\boldsymbol{\Theta}^{*}, \boldsymbol{\omega}^{*}, \delta^{*}, \boldsymbol{\tau}^{*}, \boldsymbol{\nu}^{*}, \gamma^{*}, \boldsymbol{\alpha}^{*}, \boldsymbol{\beta}^{*}\right)$ solves (31), then the solution $\left(\boldsymbol{\Theta}^{*}, \boldsymbol{\omega}^{*}\right)$ solves (23) with $\mathscr{P}=$ $\mathscr{P}_{I}$, and, conversely, if the solution $\left(\overline{\boldsymbol{\Theta}}^{*}, \overline{\boldsymbol{\omega}}^{*}\right)$ solves (23) with $\mathscr{P}=\mathscr{P}_{I}$, then $\left(\overline{\boldsymbol{\Theta}}^{*}, \overline{\boldsymbol{\omega}}^{*}, \bar{\delta}^{*}, \overline{\boldsymbol{\tau}}^{*}, \overline{\boldsymbol{\nu}}^{*}, \overline{\boldsymbol{\gamma}}^{*}, \overline{\boldsymbol{\alpha}}^{*}, \overline{\boldsymbol{\beta}}^{*}\right)$ solves (31), where $\left(\bar{\delta}^{*}, \overline{\boldsymbol{\tau}}^{*}, \overline{\boldsymbol{\nu}}^{*}\right)$ and $\left(\overline{\boldsymbol{\gamma}}^{*}, \overline{\boldsymbol{\alpha}}^{*}, \overline{\boldsymbol{\beta}}^{*}\right)$ are the optimal solutions of (28) and (30), respectively.

Proof. Let $\left(\boldsymbol{\Theta}^{*}, \boldsymbol{\omega}^{*}, \delta^{*}, \boldsymbol{\tau}^{*}, \boldsymbol{\nu}^{*}, \boldsymbol{\gamma}^{*}, \boldsymbol{\alpha}^{*}, \boldsymbol{\beta}^{*}\right)$ be a solution to (31); then, $\left(\delta^{*}, \boldsymbol{\tau}^{*}, \boldsymbol{\nu}^{*}\right)$ and $\left(\boldsymbol{\gamma}^{*}, \boldsymbol{\alpha}^{*}, \boldsymbol{\beta}^{*}\right)$ would be feasible to (28) and (30), respectively. Assuming there exists another solution $\left(\widetilde{\boldsymbol{\Theta}}^{*}, \widetilde{\boldsymbol{\omega}}^{*}\right)$ to problem $(23)$ such that $\Upsilon\left(\boldsymbol{\Theta}^{*}, \boldsymbol{\omega}^{*}\right)<\Upsilon\left(\widetilde{\boldsymbol{\Theta}}^{*}, \widetilde{\boldsymbol{\omega}}^{*}\right)$, where $\Upsilon(\Theta, \omega)=\min _{\mathbf{p r} \in \mathscr{P}_{I}}(\boldsymbol{\pi}-2 \lambda \boldsymbol{\omega})^{T} \mathbf{p r}-\bar{f}$, we can obtain $\left(\widetilde{\delta}^{*}, \widetilde{\boldsymbol{\tau}}^{*}, \widetilde{\boldsymbol{\nu}}^{*}\right)$ and $\left(\widetilde{\boldsymbol{\gamma}}^{*}, \widetilde{\boldsymbol{\alpha}}^{*}, \widetilde{\boldsymbol{\beta}}^{*}\right)$ by solving (28) and (30), respectively. It can be seen that $\left(\widetilde{\boldsymbol{\Theta}}^{*}, \widetilde{\boldsymbol{\omega}}^{*}, \widetilde{\delta}^{*}, \widetilde{\boldsymbol{\tau}}^{*}, \widetilde{\boldsymbol{\nu}}^{*}, \widetilde{\gamma}^{*}, \widetilde{\boldsymbol{\alpha}}^{*}, \widetilde{\boldsymbol{\beta}}^{*}\right)$ is also feasible to (30). Since the objective functions of (23) and (31) have the same form, it contradicts the assumption that $\left(\boldsymbol{\Theta}^{*}, \boldsymbol{\omega}^{*}, \delta^{*}, \boldsymbol{\tau}^{*}, \boldsymbol{\nu}^{*}, \boldsymbol{\gamma}^{*}, \boldsymbol{\alpha}^{*}, \boldsymbol{\beta}^{*}\right)$ is a solution to (31) due to $\Upsilon\left(\boldsymbol{\Theta}^{*}, \boldsymbol{\omega}^{*}\right)<\Upsilon\left(\widetilde{\boldsymbol{\Theta}}^{*}, \widetilde{\boldsymbol{\omega}}^{*}\right)$. Therefore, $\left(\boldsymbol{\Theta}^{*}, \boldsymbol{\omega}^{*}\right)$ is a solution to (23).

Conversely, if $\left(\overline{\boldsymbol{\Theta}}^{*}, \overline{\boldsymbol{\omega}}^{*}\right)$ is optimal to (23), then $\left(\bar{\delta}^{*}, \overline{\boldsymbol{\tau}}^{*}, \overline{\boldsymbol{\nu}}^{*}\right)$ and $\left(\bar{\gamma}^{*}, \overline{\boldsymbol{\alpha}}^{*}, \overline{\boldsymbol{\beta}}^{*}\right)$ would be solutions to (28) and (30), respectively. Obviously, $\left(\overline{\boldsymbol{\Theta}}^{*}, \overline{\boldsymbol{\omega}}^{*}, \bar{\delta}^{*}, \overline{\boldsymbol{\tau}}^{*}, \overline{\boldsymbol{\nu}}^{*}, \overline{\boldsymbol{\gamma}}^{*}, \overline{\boldsymbol{\alpha}}^{*}, \overline{\boldsymbol{\beta}}^{*}\right)$ is also a feasible solution to problem (31). If there exists a different solution $\left(\widetilde{\overline{\boldsymbol{\Theta}}}^{*}, \widetilde{\boldsymbol{\omega}}^{*}, \widetilde{\bar{\delta}}^{*}, \widetilde{\overline{\boldsymbol{\tau}}}^{*}, \widetilde{\bar{\nu}}^{*}, \widetilde{\bar{\gamma}}^{*}, \widetilde{\overline{\boldsymbol{\alpha}}}^{*}, \widetilde{\overline{\boldsymbol{\beta}}}^{*}\right)$ to (31), then from the discussion of the first part of the proof, $\left(\widetilde{\bar{\Theta}}^{*}, \widetilde{\overline{\boldsymbol{\omega}}}^{*}\right)$ is an optimal solution to (23), which contradicts the assumption that $\left(\overline{\boldsymbol{\Theta}}^{*}, \overline{\boldsymbol{\omega}}^{*}\right)$ solves (23). Hence, $\left(\overline{\boldsymbol{\Theta}}^{*}, \overline{\boldsymbol{\omega}}^{*}, \bar{\delta}^{*}, \overline{\boldsymbol{\tau}}^{*}, \overline{\boldsymbol{\nu}}^{*}, \bar{\gamma}^{*}, \overline{\boldsymbol{\alpha}}^{*}, \overline{\boldsymbol{\beta}}^{*}\right)$ solves (31).

Clearly, problem (31) is a linear program and can thus be solved efficiently. In particular, problem (31) is reduced to a stochastic optimization problem with known disruption occurrence probability when $\boldsymbol{\xi}=\bar{\xi}=\mathbf{0}$. Note that if $\boldsymbol{\xi} \geq \mathbf{0}$, we can increase the value of $\overline{\mathbf{p}}$ to attain $\underline{\boldsymbol{\xi}}<\mathbf{0}$. Hence, we can always make sure that $\underline{\boldsymbol{\xi}}<\mathbf{0}$ and $\overline{\boldsymbol{\xi}}>\mathbf{0}$ hold. The constraints $\boldsymbol{\tau} \leq \mathbf{0}$ and $\boldsymbol{\nu} \geq \mathbf{0}$ in (31) imply that the term, $\overline{\boldsymbol{\xi}}^{T} \boldsymbol{\tau}+\underline{\xi}^{T} \boldsymbol{\nu}$, is a negative reflecting the performance loss caused by the uncertainty.

4.2. Robust SCN Design Model under Ellipsoid Uncertainty. The ellipsoid uncertainty set to which the probability of the supply disruption scenario belongs is defined as follows:

$$
\begin{aligned}
\mathbf{p r} & \in \mathscr{P}_{E} \triangleq\left\{\mathbf{p r} \mid \mathbf{p r}=\overline{\mathbf{p}}+\mathbf{A} \boldsymbol{\xi}, \overline{\mathbf{p}}+\mathbf{A} \boldsymbol{\xi} \geq \mathbf{0}, \mathbf{e}^{T} \mathbf{A} \boldsymbol{\xi}\right. \\
& =0,\|\boldsymbol{\xi}\| \leq 1\}
\end{aligned}
$$

where $\overline{\mathbf{p}}$ is the most likely distribution which is the center of the ellipsoid. $\|\cdot\|$ is the Euclidean norm, and $\|\cdot\|_{*}$ is its dual representation. Due to the self-duality of Euclidean norm, we have $\|\cdot\|_{*}=\|\cdot\|$. A $\in R^{n \times n}$ is a known scaling matrix to measure the degree of the uncertainty. The constraints $\mathbf{e}^{T} \mathbf{A} \boldsymbol{\xi}=0$ and $\overline{\mathbf{p}}+\mathbf{A} \boldsymbol{\xi} \geq 0$ ensure that the vector $\mathbf{p r}$ is a probability distribution. Hence, the objective of problem (23) can be formulated as follows:

$$
\max _{\Theta, \omega} \min _{\mathbf{p r} \in \mathscr{P}_{E}}(\pi-2 \lambda \boldsymbol{\omega})^{T} \mathbf{p r}-\bar{f}
$$

Consider the following inner optimization problem of problem (33):

$$
\begin{aligned}
\min _{\mathbf{p r} \in \mathscr{P}_{E}} & \left\{(\boldsymbol{\pi}-2 \lambda \boldsymbol{\omega})^{T} \mathbf{p r}-\bar{f}\right\} \\
& =(\boldsymbol{\pi}-2 \lambda \boldsymbol{\omega})^{T} \overline{\mathbf{p}}+\mathscr{Q}^{*}(\boldsymbol{\xi})-\bar{f}
\end{aligned}
$$


where $\mathbb{Q}^{*}(\xi)$ is the optimal value of the following problem:

$$
\min _{\xi}\left\{(\boldsymbol{\pi}-2 \lambda \boldsymbol{\omega})^{T} \mathbf{A} \boldsymbol{\xi} \mid \overline{\mathbf{p}}+\mathbf{A} \boldsymbol{\xi} \geq \mathbf{0}, \mathbf{e}^{T} \mathbf{A} \boldsymbol{\xi}=\mathbf{0},\|\boldsymbol{\xi}\| \leq \mathbf{1}\right\} .
$$

It can be seen that problem (35) is a second-order cone program with the variable vector $\boldsymbol{\xi}$ and its Lagrange is defined as

$$
\begin{aligned}
\mathscr{L}(\boldsymbol{\eta}, \rho, \mu ; \boldsymbol{\xi})= & (\boldsymbol{\pi}-2 \lambda \boldsymbol{\omega})^{T} \mathbf{A} \boldsymbol{\xi}+\boldsymbol{\eta}^{T}(-\overline{\mathbf{p}}-\mathbf{A} \boldsymbol{\xi}) \\
& +\rho(\|\boldsymbol{\xi}\|-1)+\mu \mathbf{e}^{T} \mathbf{A} \boldsymbol{\xi}
\end{aligned}
$$

The Lagrange dual function with $(\boldsymbol{\eta}, \rho, \mu) \in R^{N_{S}} \times R \times R$ is given by

$$
\begin{aligned}
& g(\boldsymbol{\eta}, \rho, \mu)=\min _{\xi} \mathscr{L}(\boldsymbol{\eta}, \rho, \mu ; \boldsymbol{\xi})=\left(-\overline{\mathbf{p}}^{T} \boldsymbol{\eta}-\rho\right) \\
& -\max _{\xi}\left\{\left(-\mathbf{A}^{T}(\boldsymbol{\pi}-2 \lambda \boldsymbol{\omega})+\mathbf{A}^{T} \boldsymbol{\eta}-\mathbf{A}^{T} \mathbf{e} \mu\right)^{T} \boldsymbol{\xi}\right. \\
& -\rho\|\boldsymbol{\xi}\|\}=\left(-\overline{\mathbf{p}}^{T} \boldsymbol{\eta}-\rho\right)-\Lambda^{*}\left(-\mathbf{A}^{T}(\boldsymbol{\pi}-2 \lambda \boldsymbol{\omega})\right. \\
& \left.+\mathbf{A}^{T} \boldsymbol{\eta}-\mathbf{A}^{T} \mathbf{e} \mu\right),
\end{aligned}
$$

where $\Lambda^{*}(\mathbf{y})=\left\{0,\|\mathbf{y}\|_{*} \leq \rho ; \infty,\|\mathbf{y}\|_{*}>\rho\right\}$ is the conjugate function of $\Lambda(\xi)=\rho\|\xi\|$. Then, the Lagrange dual problem associated with problem (35) is given by

$$
\max _{\boldsymbol{\eta}, \rho, \mu} g(\boldsymbol{\eta}, \rho, \mu)=\max _{\boldsymbol{\eta}, \rho, \mu}\left\{-\overline{\mathbf{p}}^{T} \boldsymbol{\eta}-\rho \mid\left\|-\mathbf{A}^{T}(\boldsymbol{\pi}-2 \lambda \boldsymbol{\omega})+\mathbf{A}^{T} \boldsymbol{\eta}-\mathbf{A}^{T} \mathbf{e} \mu\right\| \leq \rho, \boldsymbol{\eta} \geq \mathbf{0}, \rho \geq 0\right\} .
$$

Obviously, problem (38) is a concave program. According to the characteristic of a probability distribution, there exists a disturbance vector $\boldsymbol{\xi}$ such that $\overline{\mathbf{p}}+\mathbf{A} \boldsymbol{\xi}>\mathbf{0}$, which means Slater's condition holds. Therefore, in light of the strong duality theorem of Lagrange, problem (34) is equivalent to the following maximization problem with variables $(\Theta, \omega, \boldsymbol{\eta}, \rho, \mu) \in R^{N_{d}} \times R^{N_{s}} \times R^{N_{s}} \times R \times R:$

$$
\begin{aligned}
\max _{\boldsymbol{\Theta}, \boldsymbol{\eta}, \rho, \mu} & (\boldsymbol{\pi}-2 \lambda \boldsymbol{\omega})^{T} \overline{\mathbf{p}}-\overline{\mathbf{p}}^{T} \boldsymbol{\eta}-\rho-\bar{f} \\
\text { s.t. } & \left\|-\mathbf{A}^{T}(\boldsymbol{\pi}-2 \lambda \boldsymbol{\omega})+\mathbf{A}^{T} \boldsymbol{\eta}-\mathbf{A}^{T} \mathbf{e} \mu\right\| \\
& \leq \rho, \\
\boldsymbol{\eta} & \geq \mathbf{0}, \rho \geq 0 .
\end{aligned}
$$

where $\mathscr{H}^{*}(\boldsymbol{\xi})$ is the optimal value of $\min _{\boldsymbol{\xi}}\left\{\boldsymbol{\pi}^{T} \mathbf{A} \boldsymbol{\xi} \mid \overline{\mathbf{p}}+\mathbf{A} \boldsymbol{\xi} \geq\right.$ $\left.\mathbf{0}, \mathbf{e}^{T} \mathbf{A} \boldsymbol{\xi}=0,\|\boldsymbol{\xi}\| \leq 1\right\}$ which is equivalent to the following dual program:

$$
\max _{\boldsymbol{\alpha}, \gamma, \beta}\left\{-\overline{\mathbf{p}}^{T} \boldsymbol{\alpha}-\beta \mid\left\|-\mathbf{A}^{T} \boldsymbol{\pi}+\mathbf{A}^{T} \boldsymbol{\alpha}-\mathbf{A}^{T} \mathbf{e} \gamma\right\| \leq \beta, \boldsymbol{\alpha} \geq \mathbf{0}, \beta \geq 0\right\} .
$$

Consider the following optimal problem with variables $(\boldsymbol{\Theta}, \boldsymbol{\omega}, \boldsymbol{\eta}, \rho, \mu, \boldsymbol{\alpha}, \beta, \gamma) \in R^{N_{d}} \times R^{N_{S}} \times R^{N_{S}} \times R \times R \times R^{N_{S}} \times R \times R:$

$$
\begin{array}{cl}
\underset{\substack{\boldsymbol{\Theta}, \boldsymbol{\omega}, \boldsymbol{\eta}, \boldsymbol{\rho}, \boldsymbol{c} \\
\mu, \boldsymbol{\alpha}, \beta, \gamma}}{\sin } & (\boldsymbol{\pi}-2 \lambda \boldsymbol{\omega})^{T} \overline{\mathbf{p}}-\overline{\mathbf{p}}^{T} \boldsymbol{\eta}-\rho-\bar{f} \\
\text { s.t. } & \left\|-\mathbf{A}^{T}(\boldsymbol{\pi}-2 \lambda \boldsymbol{\omega})+\mathbf{A}^{T} \boldsymbol{\eta}-\mathbf{A}^{T} \mathbf{e} \mu\right\| \leq \rho, \\
& \left\|-\mathbf{A}^{T} \boldsymbol{\pi}+\mathbf{A}^{T} \boldsymbol{\alpha}-\mathbf{A}^{T} \mathbf{e} \gamma\right\| \leq \beta, \\
& \boldsymbol{\pi}^{T} \overline{\mathbf{p}}-\overline{\mathbf{p}}^{T} \boldsymbol{\alpha}-\beta \geq \pi_{s}-\omega_{s}, \quad \forall s \in S, \\
& \boldsymbol{\eta} \geq \mathbf{0}, \boldsymbol{\alpha} \geq \mathbf{0}, \rho \geq 0, \quad \beta \geq 0, \\
& \omega_{s} \geq 0, \quad \forall s \in S,
\end{array}
$$$$
\text { Constraints (11) }-(21) \text {. }
$$

Proposition 2 shows that solving (23) under ellipsoid uncertainty is equivalent to solving (42).

Proposition 2. If the solution $\left(\Theta^{*}, \boldsymbol{\omega}^{*}, \boldsymbol{\eta}^{*}, \rho^{*}, \mu^{*}, \boldsymbol{\alpha}^{*}, \beta^{*}, \gamma^{*}\right)$ solves (42), then $\left(\Theta^{*}, \omega^{*}\right)$ solves (23) with $\mathscr{P}=\mathscr{P}_{E}$, and, conversely, if the solution $\left(\overline{\boldsymbol{\Theta}}^{*}, \overline{\boldsymbol{\omega}}^{*}\right)$ solves optimization problem (23) with $\mathscr{P}=\mathscr{P}_{E}$, then $\left(\overline{\boldsymbol{\Theta}}^{*}, \overline{\boldsymbol{\omega}}^{*}, \overline{\boldsymbol{\eta}}^{*}, \bar{\rho}^{*}, \bar{\mu}^{*}, \overline{\boldsymbol{\alpha}}^{*}, \overline{\boldsymbol{\beta}}^{*}, \bar{\gamma}^{*}\right)$ solves optimization problem (42), where $\left(\overline{\boldsymbol{\eta}}^{*}, \bar{\rho}^{*}, \bar{\mu}^{*}\right)$ and $\left(\overline{\boldsymbol{\alpha}}^{*}, \bar{\beta}^{*}, \bar{\gamma}^{*}\right)$ are the optimal solutions of (39) and (41), respectively.

The proof of Proposition 2 is omitted because it is similar to that of Proposition 1. Obviously, problem (42) is a secondorder cone program and can thus be solved efficiently. In particular, (42) is reduced to a stochastic optimization problem with known disruption occurrence probability when $\boldsymbol{\xi}=\bar{\xi}=$ 0. Similarly, it can be observed that the uncertainty of the 

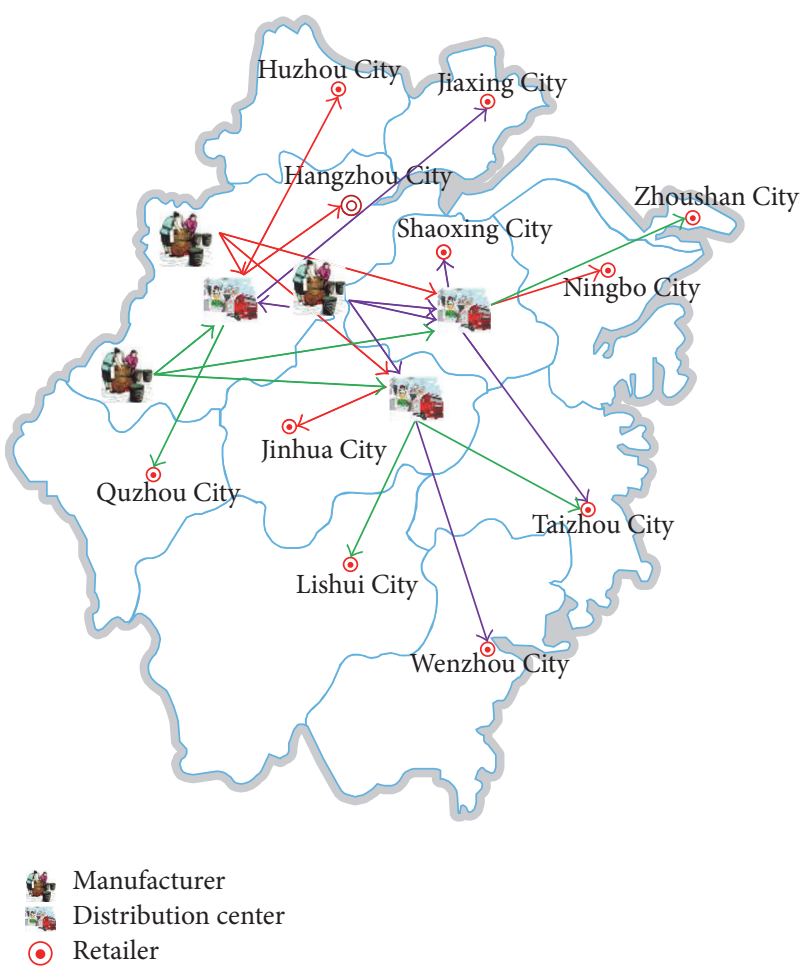

FIgURE 3: The potential supply chain network structure of CF Company.

disruption occurrence probability will lead to a performance loss (positive), that is, $\overline{\mathbf{p}}^{T} \boldsymbol{\eta}+\rho$.

\section{Case Study and Numerical Results}

To verify the effectiveness of the proposed robust SCN design models and solution approaches, we consider a threetiered supply chain network design for a real-life company in Hangzhou City of China, named CF Company, which manufactures and sales superior packed Longjing tea. The Longjing tea, produced in the surrounding mountains of the Longjing village, has efficacies of refreshing, producing saliva and slaking thirst, reducing blood lipids, lowering cholesterol, and so on. Longjing tea is usually picked and processed from March to May every year and priced from a few hundred to thousands of yuans due to the different picking time and processing technology. Due to the intense competition in local market, CF Company has to reduce the price and improve the service quality to meet the target customer group, which, however, leads to a high operation cost in return. To deal with the dilemma that the company faces, it is necessary to design an efficient and reliable supply chain network. In Figure 3, we illustrate the potential supply chain network structure of CF Company who has three available plants in Hangzhou, three potential distribution centers located at candidate locations of Hangzhou, Shaoxing, and Jiaxing, respectively, and eleven potential retailers located successively in Hangzhou, Ningbo, Wenzhou, Shaoxing, Huzhou, Jiaxing, Jinhua, Quzhou, Taizhou, Lishui, and Zhoushan.

There are twelve feasible routes in the potential supply chain network with handling costs given in Table 1. After
TABLE 1: The unit handling cost on potential routes.

\begin{tabular}{lccc}
\hline Potential route & $\begin{array}{c}\text { Unit handling } \\
\text { cost }\left(H_{p r}\right)\end{array}$ & $\begin{array}{c}\text { Potential } \\
\text { route }\end{array}$ & $\begin{array}{c}\text { Unit handling } \\
\text { cost }\left(H_{p r}\right)\end{array}$ \\
\hline$r_{1-1-1}$ & 815.1 & $r_{2-2-9}$ & 815.4 \\
$r_{1-1-5}$ & 815.3 & $r_{2-3-3}$ & 815.5 \\
$r_{1-2-2}$ & 815.2 & $r_{3-1-8}$ & 815.9 \\
$r_{1-3-7}$ & 815.3 & $r_{3-2-11}$ & 815.8 \\
$r_{2-1-6}$ & 815.3 & $r_{3-3-9}$ & 815.6 \\
$r_{2-2-4}$ & 815.2 & $r_{3-3-10}$ & 815.2 \\
\hline
\end{tabular}

$r_{i-j-m}$ dentoes a route starting from manufacturer $i$ to retailer $m$ by passing through distribution center $j$.

TABLE 2: The distribution of the demand for each market.

\begin{tabular}{lc}
\hline Market & Distribution \\
\hline (1) Hangzhou & $D_{1} \sim N\left(E\left(D_{1}\right)=700, \sigma\left(D_{1}\right)=5\right)$ \\
(2) Ningbo & $D_{2} \sim N\left(E\left(D_{2}\right)=700, \sigma\left(D_{2}\right)=5\right)$ \\
(3) Wenzhou & $D_{3} \sim N\left(E\left(D_{3}\right)=600, \sigma\left(D_{3}\right)=5\right)$ \\
(4) Shaoxing & $D_{4} \sim N\left(E\left(D_{4}\right)=500, \sigma\left(D_{4}\right)=5\right)$ \\
(5) Huzhou & $D_{5} \sim N\left(E\left(D_{5}\right)=300, \sigma\left(D_{5}\right)=5\right)$ \\
(6) Jiaxing & $D_{6} \sim N\left(E\left(D_{6}\right)=300, \sigma\left(D_{6}\right)=5\right)$ \\
(7) Jinhua & $D_{7} \sim N\left(E\left(D_{7}\right)=600, \sigma\left(D_{7}\right)=5\right)$ \\
(8) Quzhou & $D_{8} \sim N\left(E\left(D_{8}\right)=200, \sigma\left(D_{8}\right)=4\right)$ \\
(9) Taizhou & $D_{9} \sim N\left(E\left(D_{9}\right)=500, \sigma\left(D_{9}\right)=5\right)$ \\
(10) Lishui & $D_{10} \sim N\left(E\left(D_{10}\right)=200, \sigma\left(D_{10}\right)=4\right)$ \\
(11) Zhoushan & $D_{11} \sim N\left(E\left(D_{11}\right)=220, \sigma\left(D_{11}\right)=5\right)$ \\
\hline
\end{tabular}

a series of processes such as picking, spreading, fixing, shaping, and drying, the production of Longjing tea is completed and then packed into finished products $(500 \mathrm{~g}$ per box). The unit prices of the product for all markets are 965, 965, 966, 965, 965, 965, 965, 966, 965, 966, and 966, respectively. The unit shortage cost for unmet demand is 101 and the unit salvage value for residual product is 386 . The maximum production capacity of each manufacture is considered as 2500, 2200, and 1200, respectively. The fixed costs for locating distribution centers at candidate locations of Hangzhou, Shaoxing, and Jiaxing are 23120, 19884, and 18888, respectively. The fixed costs of locating retailers for the abovementioned 11 markets are 5780, 5676, 5517, 4971, $4957,4998,4722,4825,5221,4830$, and 4943. The budget for establishing the facilities is 120000 . The random demand of each market is assumed to follow Gaussian distribution (see Table 2). Due to confidentiality, all the data used in this paper are processed but resemble the data structure of CF Company.

We consider four possible disruption scenarios due to the manufacturers' facilities impairments and connection links interruptions. In the first scenario, all twelve potential routes are available which means there is no disruption occurring in the supply chain. The second and the third scenarios are described as only the second and the third facility break down, respectively. And in the fourth scenario, the second and the third facility fail simultaneously. The probability of each scenario to occur is only known to belong 


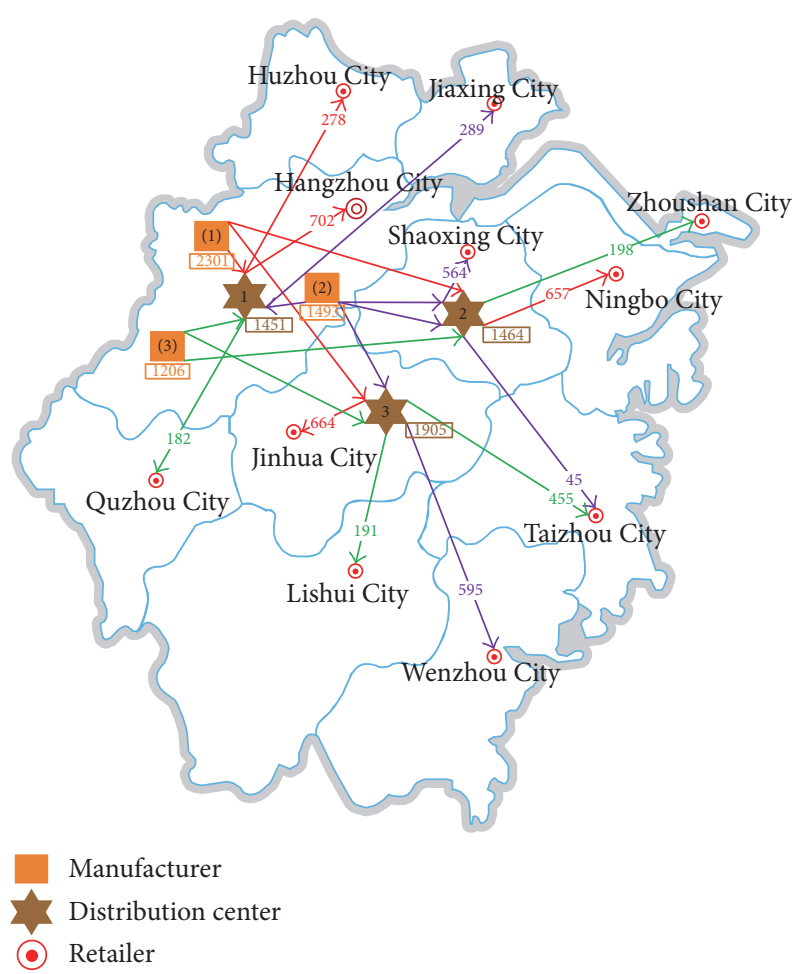

FIGURE 4: Network structure and operation strategies under $\overline{\mathbf{p}}$ in the first scenario.

to a specific uncertainty set, where the most likely distribution is estimated to be $\overline{\mathbf{p}}=(0.6875,0.0500,0.2500,0.0125)^{T}$.

We break the range of each target market into ten intervals and assume $\overline{\mathbf{p}}$ is the real disruption occurrence probability distribution for the purpose of comparing the robust solution with the optimal one. Models (31) and (42) are solved with LINGO 11.00 software and the runs are performed on an Intel Core with 2 Duo CPU at $2.2 \mathrm{GHz}$. The solutions are obtained within two minutes approximately.

5.1. Results under $\overline{\mathbf{p}}$. By solving (22b) with $\mathbf{p r}=\overline{\mathbf{p}}$ and $\lambda=2$, we obtain the optimal objective function value of 375647.3, and the corresponding optimal network structures under different scenarios are displayed in Figures 4-7, where the numbers in the boxes and lines represent the manufacturers' production quantity, distribution centers' transfer amount, and markets sales volume. From Figure 4, we find that the yields of three manufactures in the first scenario are 2301, 1493, and 1206, respectively. All the potential markets are selected and the total quantities shipped to these markets are 5000. Figure 5 (Figure 6) shows that the total yield, that is, 3404 (4184), does not reach the maximum capacity in the second (third) scenario. Compared with the first scenario, we find the production quantity in the second or third scenario is less than that in the first one due to the impairments of the manufacturers' facilities, which results in the unmet markets such as 3, 4, and 6 under the second scenario and markets 8 , 10 , and 11 under the third scenario. Considering that only the first facility can be usable, we find that only a few markets are selected in the fourth scenario, with the total sales volume of 2292 (see Figure 7).

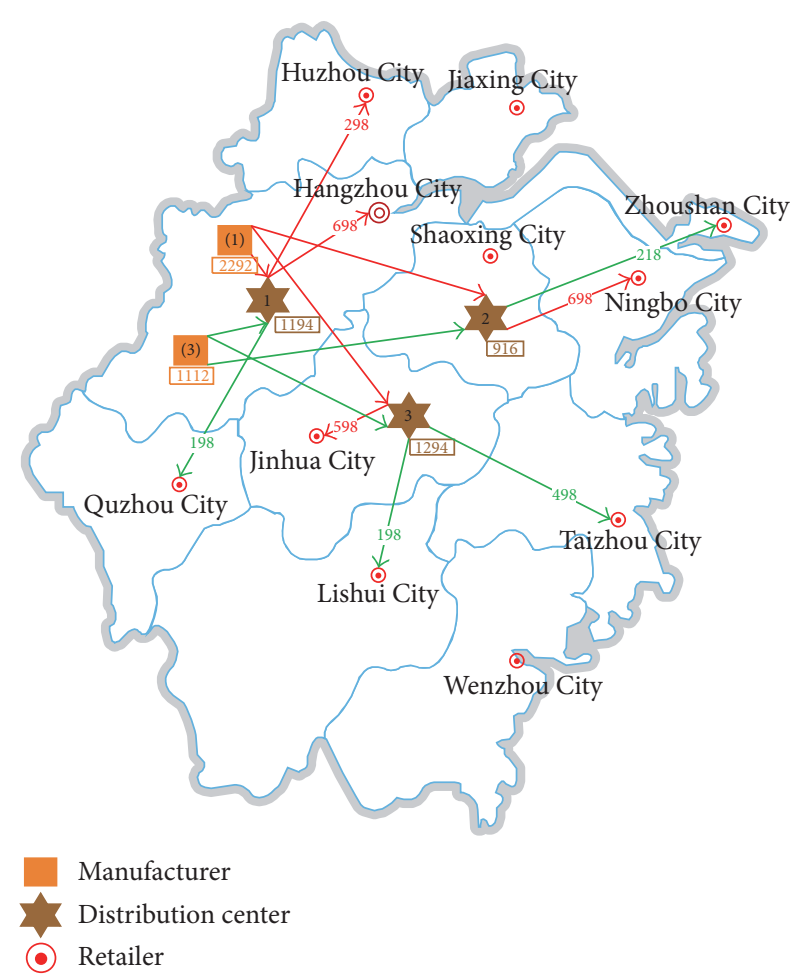

FIGURE 5: Network structure and operation strategies under $\overline{\mathbf{p}}$ in the second scenario.

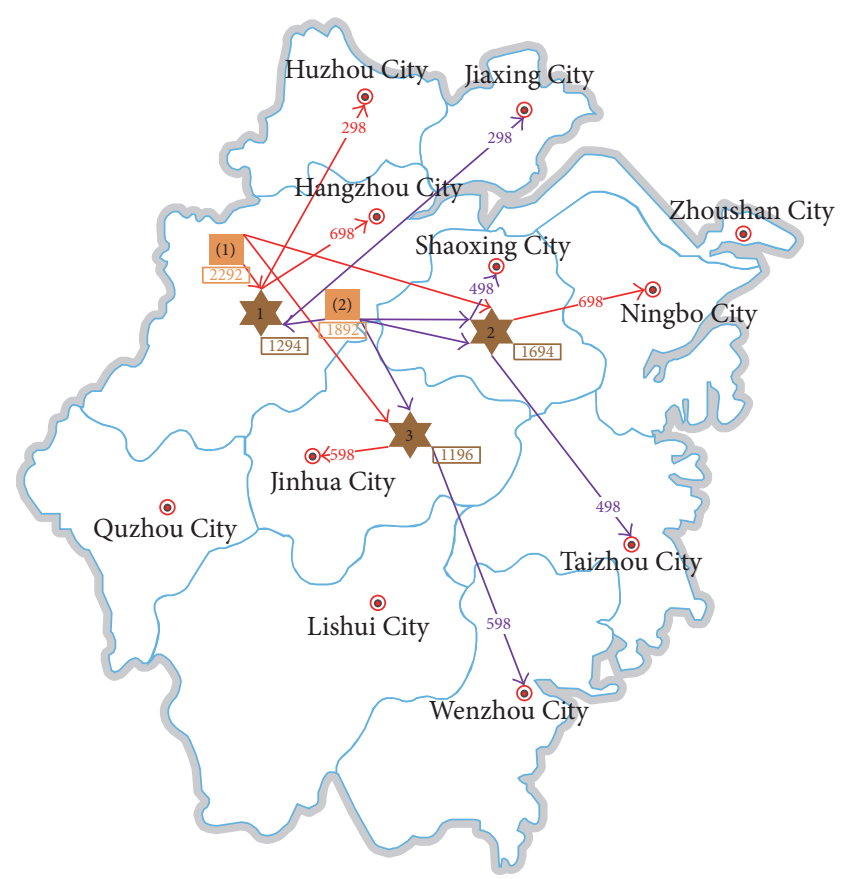

Manufacturer

Distribution center

- Retailer

Figure 6: Network structure and operation strategies under $\overline{\mathbf{p}}$ in the third scenario. 


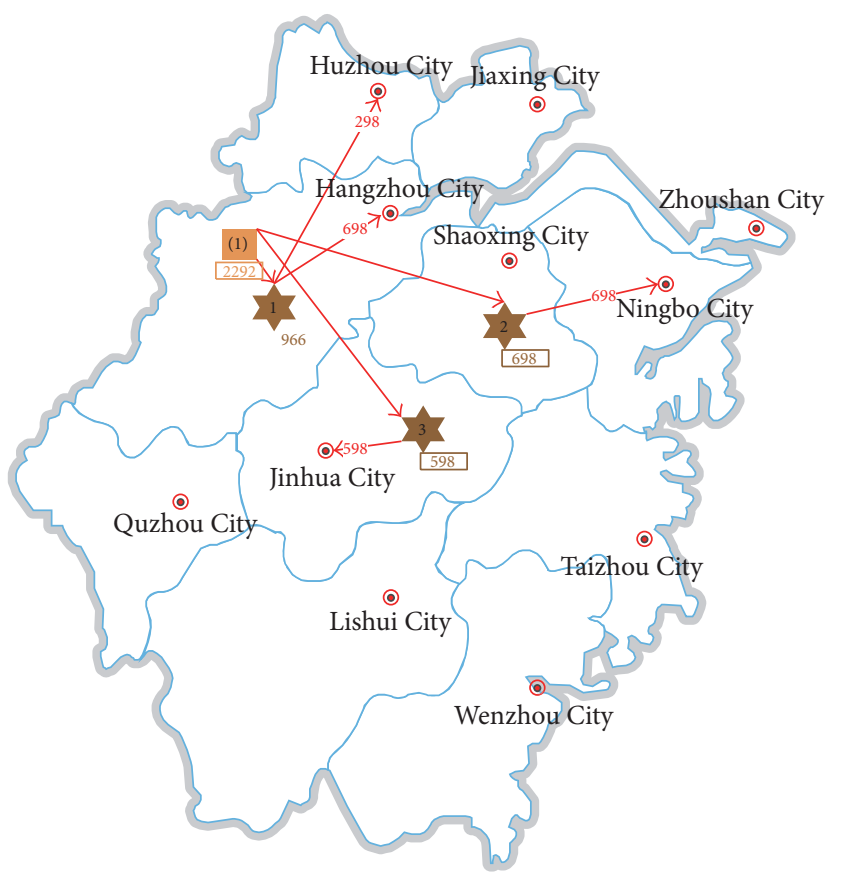

Manufacturer

2 Distribution center

(-) Retailer

Figure 7: Network structure and operation strategies under $\overline{\mathbf{p}}$ in the fourth scenario.

5.2. Results under Box Uncertainty. In order to test the proposed SCN design model and the solution approach under box uncertainty, let $\boldsymbol{\xi}=g \cdot \mathbf{K}, \mathbf{K} \in[\underline{\mathbf{K}}, \overline{\mathbf{K}}], \underline{\mathbf{K}}=$ $(-1,-0.2,-0.6,-0.05)^{T}, \overline{\mathbf{K}}=(1,0.2,0.6,0.05)^{T}, g=0.02$, and $\lambda=2$. By solving problem (31), we obtain the objective function value of 310826.5 . However, when the solutions derived from (31) are applied to the real distribution $\overline{\mathbf{p}}$, the corresponding performance turns into 324167.4. Comparing with the optimal value of 375647.3 , it can be found that the uncertainty in disruption scenario occurrence probability will inevitably lead to the performance loss ((375647.3 $324167.4) / 375647.3=13.7 \%)$. Furthermore, the loss can be regarded as the maximum cost for decision-makers who are willing to pay to obtain the complete disruption information. Interestingly, by observing the network structures under box uncertainty, we find that only the SCN design plan in the first scenario is different from that in the real distribution $\overline{\mathbf{p}}$ (see Figure 8 ). We can thus conclude that the proposed robust SCN design model and solution approach perform well under uncertainty. In particular, the network structure and corresponding operation strategies are consistent with the optimal case when the supply disruption does occur in the supply chain.

To further investigate the effectiveness of the proposed robust optimization approach, some of the probability distributions are generated according to (24) with $\overline{\mathbf{p}}=$ $(0.6875,0.0500,0.2500,0.0125)^{T}$, and all of them are considered as the real distributions. The calculation results are displayed in Table 3 where the numbers in the third column

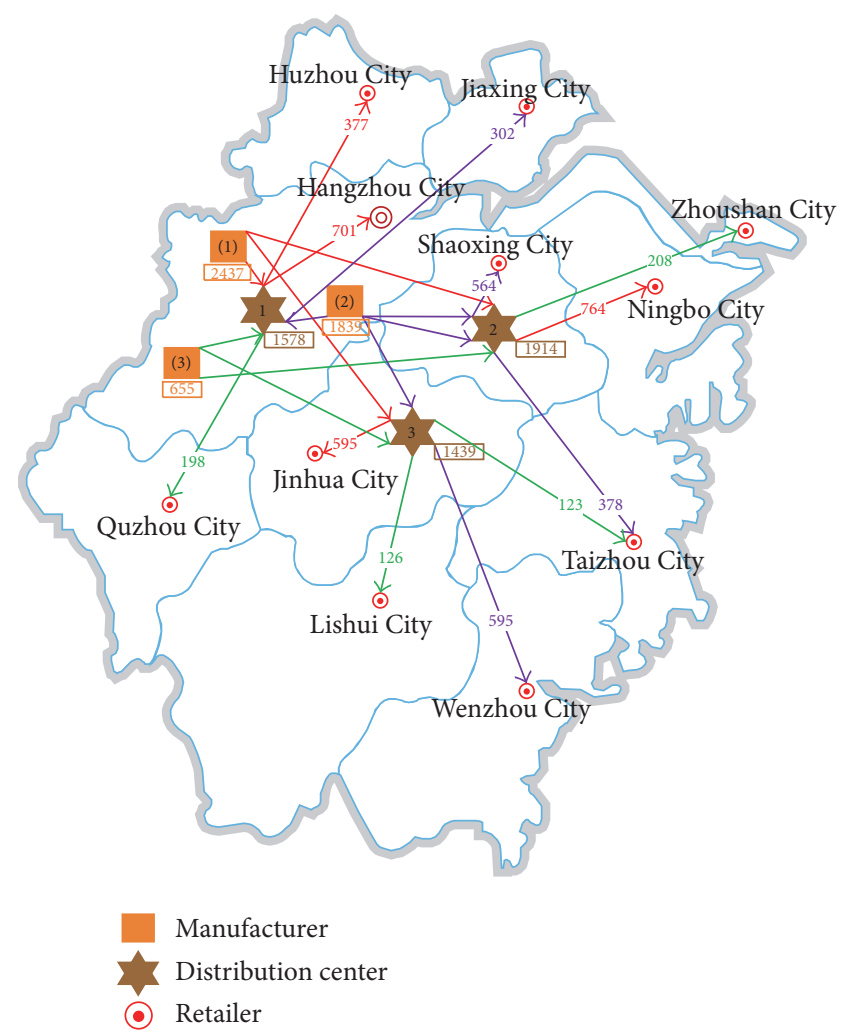

FIGURE 8: Network structure and operation strategies under box certainty in the first scenario.

are obtained by applying the robust solutions to each of the real distributions generated. From Table 3, we find that the performance loss ratios change around $13.70 \%$ with relatively small amplitude, which indicates the proposed solution approach in this paper is robust to deal with uncertainty in disruption scenario probability distribution.

Since the variability weight $\lambda$ places an important role in measuring the deviation between the robust performance for any scenario realization and the optimal one, more experiments are executed to observe the changing tendencies of the objective performances under different $\lambda$ and the degree of uncertainty expressed with a parameter $g$ as well. We obtain the results shown in Figure 9. From Figure 9, we find that, for a given $\lambda$ or $g$, the performance is decreasing with the degree of the uncertainty or the variability weight $\lambda$. However, the lower the degree of the uncertainty, the smaller the decreasing amplitude, which implies the supply chain manager should gather more information to improve the forecasting precision of disruption scenario probability.

5.3. Results under Ellipsoid Uncertainty. When the probabilities that supply disruption scenarios occur belong to the ellipsoid uncertainty set defined in (32), we do the similar numerical simulations as in Section 5.2. Let $\mathbf{A}=h$. $\mathbf{E}$, where $\mathbf{E}$ is an identity matrix, $h=0.02$, and $\lambda=$ 2 . By solving (42), we obtain the objective function value of 331352.9. The solutions derived from (42) produce a performance of 341832.6 when applying them to the real distribution $\overline{\mathbf{p}}$, which leads to the performance loss ratio as 
TABLE 3: Comparisons under different probability distributions under box uncertainty.

\begin{tabular}{lccc}
\hline Scenario probability distribution & $\begin{array}{c}\text { Optimal objective } \\
\text { function value }\end{array}$ & $\begin{array}{c}\text { Objective function value when applying } \\
\text { robust solutions to a real distribution }\end{array}$ & $\begin{array}{c}\text { Performance loss ratio } \\
(0.7295,0.0500,0.2100,0.0105)\end{array}$ \\
$(0.7085,0.0500,0.2300,0.0115)$ & 379422.0 & 319096.0 & $15.90 \%$ \\
$(0.6875,0.0500,0.2500,0.0125)$ & 375647.3 & 321631.7 & $14.81 \%$ \\
$(0.6665,0.0500,0.2700,0.0135)$ & 373759.9 & 324167.4 & $13.70 \%$ \\
$(0.6455,0.0500,0.2900,0.0145)$ & 371872.6 & 326703.0 & $12.59 \%$ \\
\hline
\end{tabular}

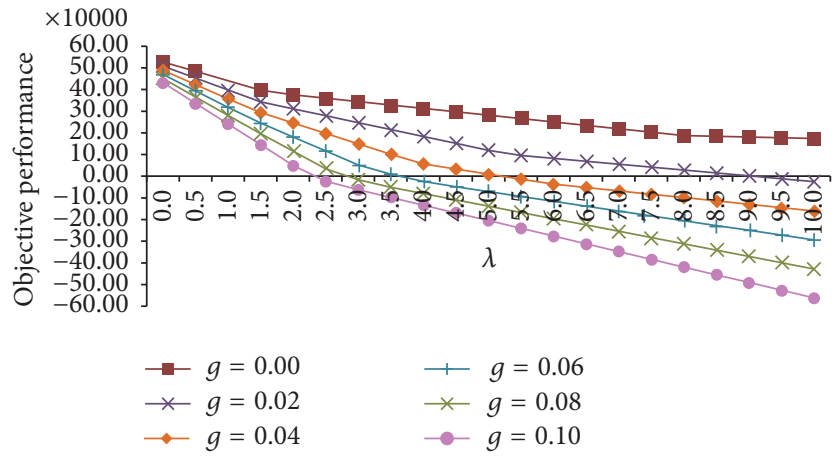

FIGURE 9: Sensitivity analysis on $\lambda$ and $g$ under box uncertainty.

9.00\% ((375647.3 - 341832.6)/375647.3). Similarly, we also find that only the network structure and the corresponding operation strategies in the first scenario (see Figure 10) are different from the optimal case.

Similarly, in order to further examine the effectiveness of the proposed robust optimization approach under ellipsoid uncertainty, some more probability distributions are generated according to (32) with $\overline{\mathbf{p}}=(0.6875,0.0500,0.2500$, $0.0125)^{T}$ and regarded as the real distributions. Following the same calculation as above, we obtain the results displayed in Table 4. From Table 4, we find that the performance loss ratios change closely around $9.00 \%$, which validates the effectiveness of the proposed solution approach in dealing with the distributional uncertainty. Moreover, the performance changing tendencies under different variability weight $\lambda$ and the degree of the uncertainty $h$ are investigated and the results are shown in Figure 11 which reveals the similar relationships as those found in Figure 9.

\section{Conclusions}

In this paper, we develop a robust optimization model for designing a supply chain network including manufactures, distribution centers, and retailers under both demand uncertainty and supply disruption. For uncertain market demand, it is assumed to be random and follow a known distribution, and a piecewise linearization method is used to transform the nonlinear model caused by stochastic demand into a linear one. For supply disruption, it is described by a series

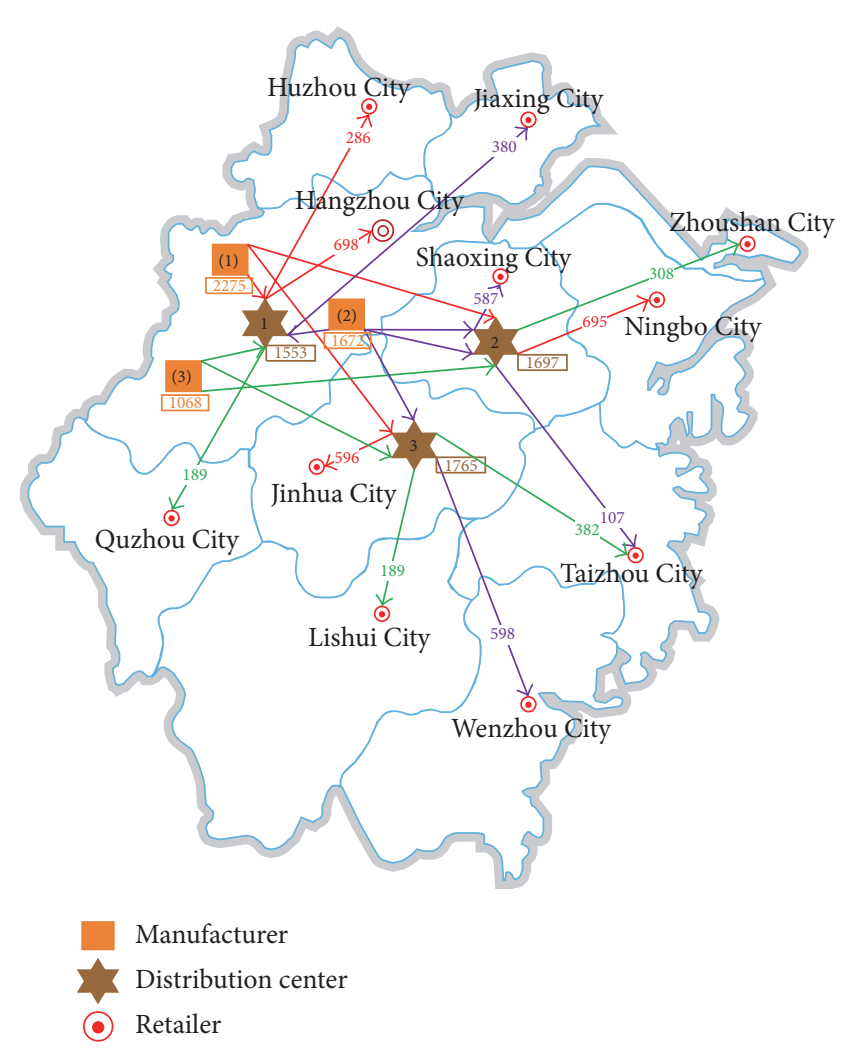

FIGURE 10: Network structure and operation strategies under ellipsoid uncertainty in the first scenario.

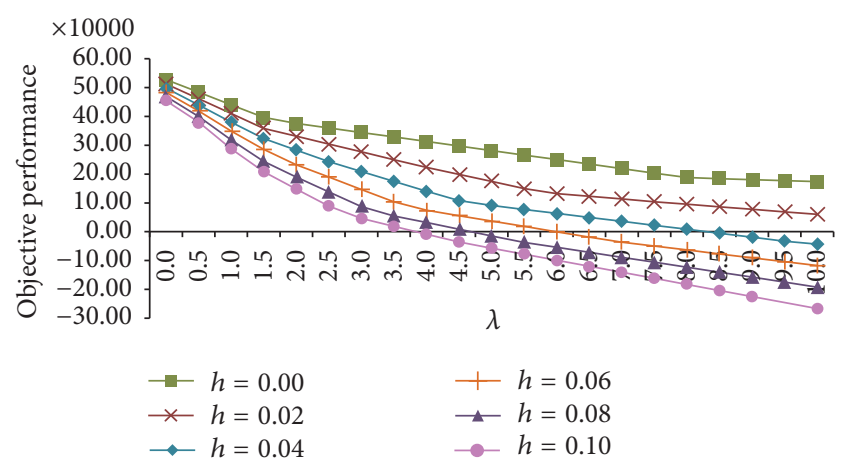

FIGURE 11: Sensitivity analysis on $\lambda$ and $h$ under ellipsoid uncertainty. 
TABLE 4: Comparisons under different probability distributions under ellipsoid uncertainty.

\begin{tabular}{lccc}
\hline Scenario probability distribution & $\begin{array}{c}\text { Optimal objective function } \\
\text { value }\end{array}$ & $\begin{array}{c}\text { Objective function value when applying } \\
\text { robust solutions to a real distribution }\end{array}$ & $\begin{array}{c}\text { Performance loss ratio } \\
(0.7295,0.0500,0.2100,0.0105)\end{array}$ \\
$(0.7085,0.0500,0.2300,0.0115)$ & 379422.0 & 337840.5 & $10.96 \%$ \\
$(0.6875,0.0500,0.2500,0.0125)$ & 375647.3 & 339836.5 & $9.99 \%$ \\
$(0.6665,0.0500,0.2700,0.0135)$ & 373759.9 & 341832.7 & $9.00 \%$ \\
$(0.6455,0.0500,0.2900,0.0145)$ & 371872.6 & 343828.7 & $8.01 \%$ \\
\hline
\end{tabular}

of scenarios with unknown probabilities and the corresponding robust counterparts are presented under both box and ellipsoid uncertainty. Through mathematical propositions, we transform the robust SCN design models into tractable linear program under box uncertainty and into second-order cone program under ellipsoid uncertainty.

The proposed model and solution approach are evaluated in a real case to examine their effectiveness. The results show that the SCN designed by our approach perform well under the uncertainty in supply disruption scenario probability. In particular, the lower the degree of the uncertainty, the better the operation performance of the supply chain network. Our robust supply chain design model can be extended to multiperiod case by incorporating an inventory management policy. In addition, another interesting direction for future research is to consider other risk measurements such as conditional value at risk $(\mathrm{CVaR})$ and mean $\mathrm{CVaR}$ to measure the performance under uncertainty which would enrich our model.

\section{Notations}

Sets

I: Set of manufacturers, indexed by $i$, $i \in I=\left\{1,2, \ldots, N_{I}\right\}$

$J$ : Set of potential distribution centers, indexed by $j, m \in M=\left\{1,2, \ldots, N_{M}\right\}$

$M$ : Set of markets or retailers, indexed by $m$, $m \in M=\left\{1,2, \ldots, N_{M}\right\}$

$P$ : Set of products, indexed by $p$, $p \in P=\left\{1,2, \ldots, N_{P}\right\}$

$S: \quad$ Set of disruption scenarios, indexed by $s$, $s \in S=\left\{1,2, \ldots, N_{S}\right\}$

$R$ : Set of routes starting from manufacturers, passing through distribution centers, and ending at retailers, indexed by $r$, $r \in R=\left\{1,2, \ldots, N_{R}\right\}$

$R^{i}$ : Set of routes starting from manufacturer $i$

$R^{j}$ : Set of routes passing through distribution center $j$

$R^{m}$ : Set of routes shipped to market $m$.

\section{Parameters}

$G_{j}$ : Fixed cost of establishing distribution center $j$
$L_{m}:$ Fixed cost of developing market $m$

$D_{m p}$ : Demand of product $p$ in market $m$, which is a random variable with the mean $E\left[D_{m p}\right]$ and the cumulative distribution function $F\left(D_{m p}\right)$

$U_{i p}$ : Capacity of manufacturer $i$ for product $p$

$y: \quad$ Cost budget for establishing facilities in supply chain network

$P_{m p}:$ Unit sales price of product $p$ in market $m$

$V_{m p}$ : Unit salvage value of unsold product $p$ in market $m$

$C_{m p}$ : Unit shortage cost of the unmet demand for product $p$ in market $m$

$H_{p r}$ : Unit handling cost for product $p$ along route $r$, including unit production cost, unit shipping cost, and unit storage cost

$p_{s}: \quad$ Probability of disruption occurrence of scenario $s$.

\section{Decision Variables}

$Q_{p s r}$ : Amount of product $p$ shipped along the route $r$ in scenario $s$

$\kappa_{j}$ : Binary variable equal to 1 if a distribution center is established in location $j$ and 0 otherwise

$\zeta_{m}: \quad$ Binary variable equal to 1 if market $m$ is opened and 0 otherwise

$B_{s r}:$ Binary variable equal to 1 if route is available in scenario $s$ and 0 otherwise.

\section{Competing Interests}

The authors declare that they have no competing interests.

\section{Acknowledgments}

This research is partly supported by the National Natural Science Foundation of China (no. 71372186) and Fundamental Research Funds for the Central Universities (N150604005).

\section{References}

[1] W. Klibi and A. Martel, "The design of robust value-creating supply chain networks," OR Spectrum, vol. 35 , no. 4, pp. 867903, 2013. 
[2] A. Nagurney and L. S. Nagurney, "Sustainable supply chain network design: a multicriteria perspective," International Journal of Sustainable Engineering, vol. 3, no. 3, pp. 189-197, 2010.

[3] M. Bashiri, H. Badri, and J. Talebi, "A new approach to tactical and strategic planning in production-distribution networks," Applied Mathematical Modelling, vol. 36, no. 4, pp. 1703-1717, 2012.

[4] S. Mahdi, C. G. Marti, and S. Nilay, "Supply chain network design and operation: systematic decision-making for centralized, distributed, and mobile biofuel production using mixed integer linear programming (MILP) under uncertainty," Biomass and Bioenergy, vol. 81, no. 2, pp. 401-414, 2015.

[5] M. Jeihoonian, M. K. Zanjani, and M. Gendreau, "Accelerating Benders decomposition for closed-loop supply chain network design: case of used durable products with different quality levels," European Journal of Operational Research, vol. 251, no. 3, pp. 830-845, 2016.

[6] W. Klibi, A. Martel, and A. Guitouni, "The design of robust value-creating supply chain networks: a critical review," European Journal of Operational Research, vol. 203, no. 2, pp. 283293, 2010.

[7] M. Tokman and L. S. Beitelspacher, "Supply chain networks and service-dominant logic: suggestions for future research," International Journal of Physical Distribution and Logistics Management, vol. 41, no. 7, pp. 717-726, 2011.

[8] R. Z. Farahani, S. Rezapour, T. Drezner, and S. Fallah, "Competitive supply chain network design: an overview of classifications, models, solution techniques and applications," Omega, vol. 45, no. 2, pp. 92-118, 2014.

[9] M. T. Melo, S. Nickel, and F. Saldanha-da-Gama, "Facility location and supply chain management-a review," European Journal of Operational Research, vol. 196, no. 2, pp. 401-412, 2009.

[10] M. Esmaeilikia, B. Fahimnia, J. Sarkis, K. Govindan, A. Kumar, and J. Mo, "Tactical supply chain planning models with inherent flexibility: definition and review," Annals of Operations Research, vol. 23, no. 1, pp. 1-21, 2014.

[11] C. S. Tang, "Perspectives in supply chain risk management," SSRN Electronic Journal, vol. 103, no. 2, pp. 451-488, 2006.

[12] N. S. Sadghiani, S. A. Torabi, and N. Sahebjamnia, "Retail supply chain network design under operational and disruption risks," Transportation Research Part E: Logistics \& Transportation Review, vol. 75, no. 1, pp. 95-114, 2015.

[13] J. M. Mulvey, R. J. Vanderbei, and S. A. Zenios, "Robust optimization of large-scale systems," Operations Research, vol. 43, no. 2, pp. 264-281, 1995.

[14] R. Babazadeh and J. Razmi, "A robust stochastic programming approach for agile and responsive logistics under operational and disruption risks," International Journal of Logistics Systems and Management, vol. 13, no. 4, pp. 458-482, 2012.

[15] A. Baghalian, S. Rezapour, and R. Farahani, "Robust supply chain network design with service level against disruptions and demand uncertainties: a real-life case," European Journal of Operational Research, vol. 227, no. 1, pp. 199-215, 2013.

[16] A. Jabbarzadeh, B. Fahimnia, and S. Seuring, "Dynamic supply chain network design for the supply of blood in disasters: a robust model with real world application," Transportation Research Part E: Logistics \& Transportation Review, vol. 70, pp. 225-244, 2014.
[17] K.-Y. Jeong, J.-D. Hong, and Y. Xie, "Design of emergency logistics networks, taking efficiency, risk and robustness into consideration," International Journal of Logistics Research and Applications, vol. 17, no. 1, pp. 1-22, 2014.

[18] M. Fattahi and K. Govindan, "Investigating risk and robustness measures for supply chain network design under demand uncertainty: a case study of glass supply chain," International Journal of Production Economics, 2015.

[19] A. Hasani, S. H. Zegordi, and E. Nikbakhsh, "Robust closedloop supply chain network design for perishable goods in agile manufacturing under uncertainty," International Journal of Production Research, vol. 50, no. 16, pp. 4649-4669, 2012.

[20] S. Zokaee, A. Jabbarzadeh, B. Fahimnia et al., "Robust supply chain network design: an optimization model with real world application," Annals of Operations Research, vol. 21, no. 2, pp. $1-30,2014$.

[21] A. L. Soyster, "Convex programming with set-inclusive constraints and applications to inexact linear programming," Operations Research, vol. 21, no. 5, pp. 1154-1157, 1973.

[22] A. Ben-Tal and A. Nemirovski, "Robust solutions of linear programming problems contaminated with uncertain data," Mathematical Programming, Series B, vol. 88, no. 3, pp. 411-424, 2000.

[23] D. Bertsimas and M. Sim, “The price of robustness," Operations Research, vol. 52, no. 1, pp. 35-53, 2004.

[24] A. Ben-Tal, A. Goryashko, E. Guslitzer, and A. Nemirovski, "Adjustable robust solutions of uncertain linear programs," Mathematical Programming, vol. 99, no. 2, pp. 351-376, 2004.

[25] A. Ben-Tal, B. D. Chung, S. R. Mandala, and T. Yao, "Robust optimization for emergency logistics planning: risk mitigation in humanitarian relief supply chains," Transportation Research Part B: Methodological, vol. 45, no. 8, pp. 1177-1189, 2011.

[26] M. S. Pishvaee, M. Rabbani, and S. A. Torabi, "A robust optimization approach to closed-loop supply chain network design under uncertainty," Applied Mathematical Modelling, vol. 35, no. 2, pp. 637-649, 2011.

[27] S. M. Hatefi and F. Jolai, "Robust and reliable forward-reverse logistics network design under demand uncertainty and facility disruptions," Applied Mathematical Modelling, vol. 38, no. 9-10, pp. 2630-2647, 2014.

[28] A. Hasani, S. H. Zegordi, and E. Nikbakhsh, "Robust closedloop global supply chain network design under uncertainty: the case of the medical device industry," International Journal of Production Research, vol. 53, no. 5, pp. 1596-1624, 2015.

[29] A. A. Akbari and B. Karimi, "A new robust optimization approach for integrated multi-echelon, multi-product, multiperiod supply chain network design under process uncertainty," International Journal of Advanced Manufacturing Technology, vol. 79, no. 1-4, pp. 229-244, 2015.

[30] A. Hasani and A. Khosrojerdi, "Robust global supply chain network design under disruption and uncertainty considering resilience strategies: a parallel memetic algorithm for a reallife case study," Transportation Research Part E: Logistics and Transportation Review, vol. 87, no. 1, pp. 20-52, 2016.

[31] S. Rezapour, J. K. Allen, T. B. Trafalis et al., "Robust supply chain network design by considering demand-side uncertainty and supply-side disruption," in Proceedings of the ASME International Design Engineering Technical Conference, V03AT03A030, Portland, Ore, USA, August 2013. 
[32] N. Azad, G. K. D. Saharidis, H. Davoudpour, H. Malekly, and S. A. Yektamaram, "Strategies for protecting supply chain networks against facility and transportation disruptions: an improved Benders decomposition approach," Annals of Operations Research, vol. 210, no. 1, pp. 125-163, 2013.

[33] C. S. Yu and H. L. Li, "A robust optimization model for stochastic logistic problems," International Journal of Production Economics, vol. 64, no. 1-3, pp. 385-397, 2000. 

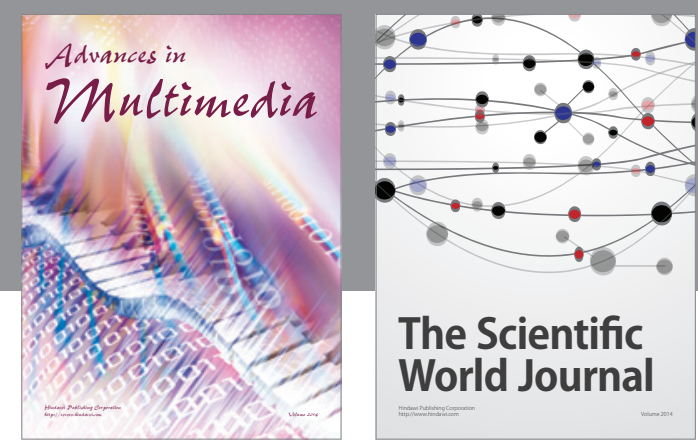

The Scientific World Journal
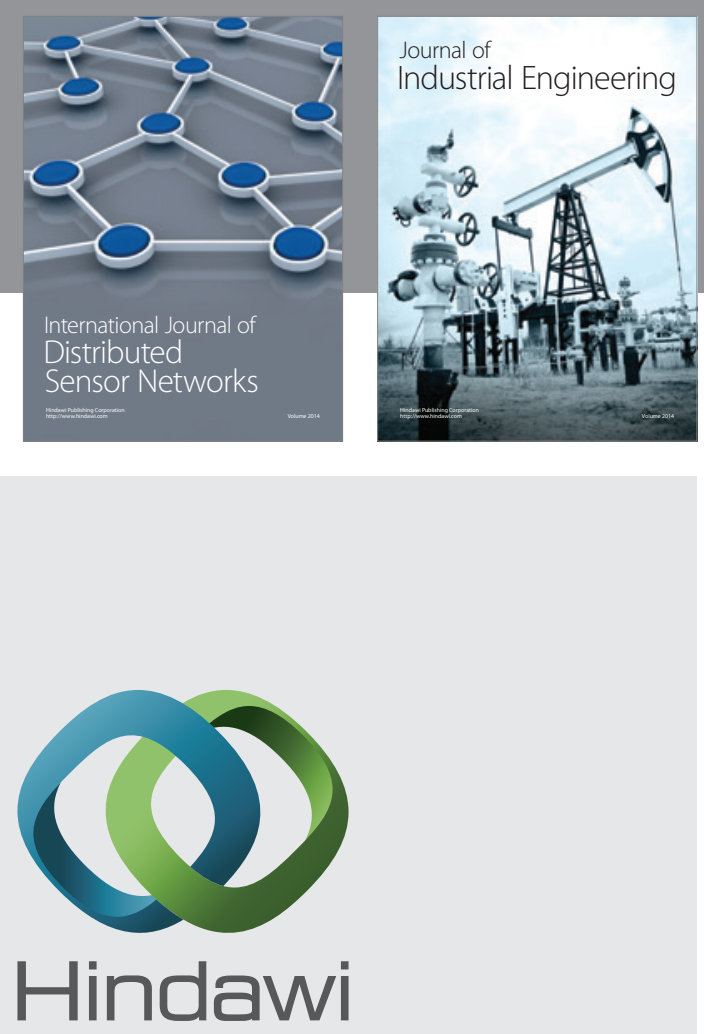

Submit your manuscripts at

http://www.hindawi.com

\section{Computer Networks} and Communications
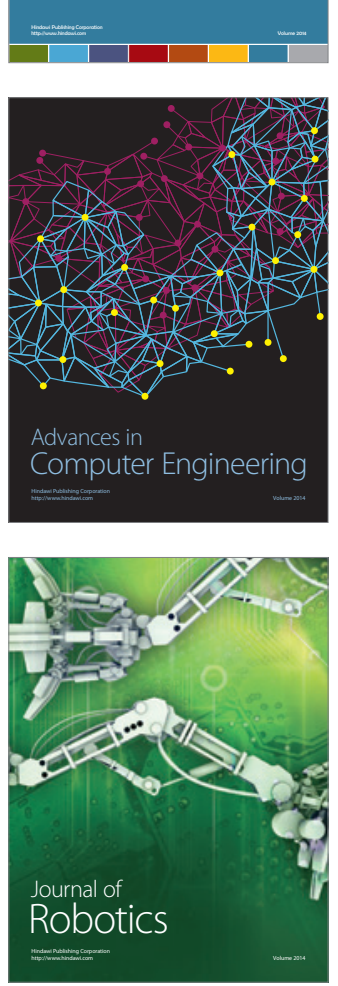
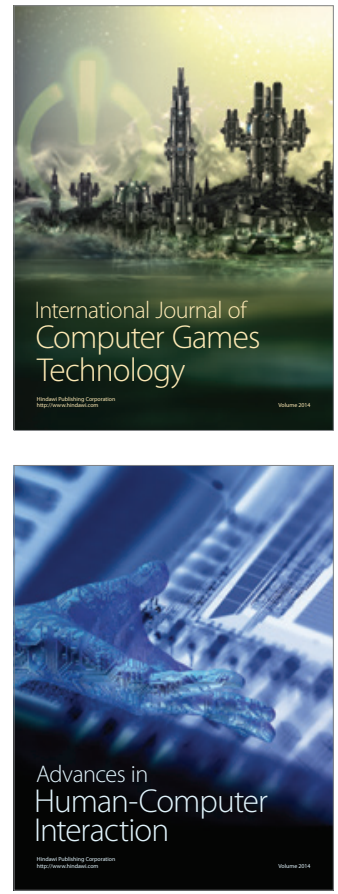
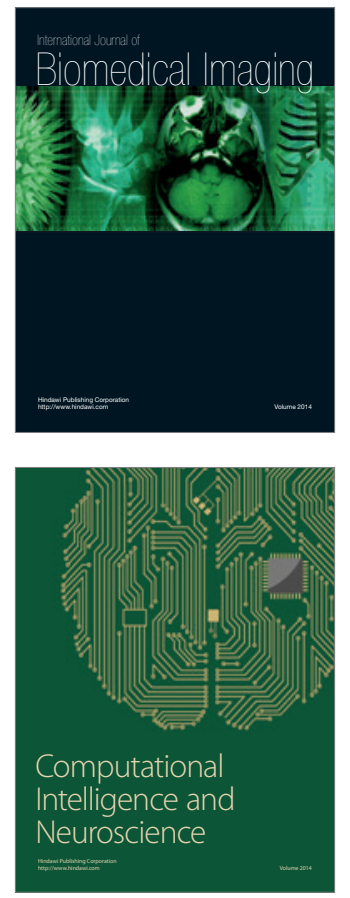
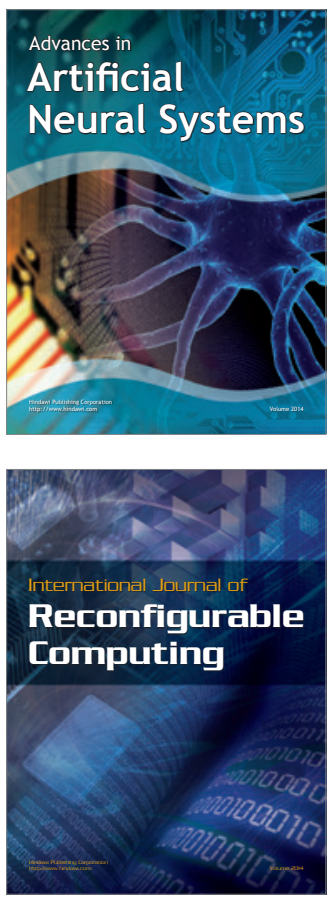
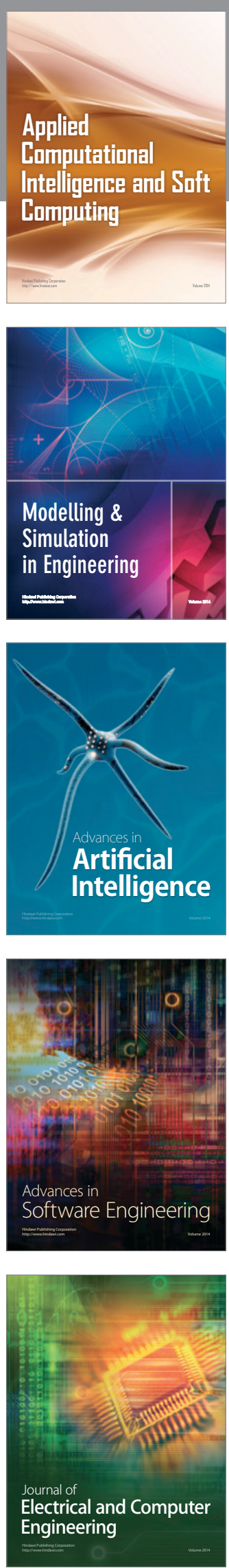